\title{
LncRNA MIR155HG functions as a ceRNA for inhibition of lung adenocarcinoma growth and prediction of prognosis
}

Jing Wang, Jie Li

Department of Thoracic Surgery, Beijing Friendship Hospital, Capital Medical University, Beijing, China

Submitted: 4 August 2020

Accepted: 4 December 2020

Arch Med Sci

DOI: https://doi.org/10.5114/aoms/131198

Copyright $\odot 2020$ Termedia \& Banach

\section{Abstract}

Introduction: Long non-coding RNAs (IncRNAs) functioning as competing endogenous RNAs (ceRNAs) play critical roles in tumour progression. However, prognosis-related ceRNA networks in lung adenocarcinoma (LUAD) have not been well characterised.

Material and methods: LUAD datasets were downloaded from the TCGA database, and the patients were divided into metastasis and non-metastasis groups. The differential expression of IncRNAs (DELs), miRNAs (DEMs), and mRNAs (DEGs) was analysed using the Limma package. Next, interactions between miRNA, IncRNA, and mRNA were predicted by miRcode, miRTarBase, miRDB, and TargetScan. The ceRNA network was constructed based on these interactions using Cytoscape software. DEG enrichment analysis was performed by GO and KEGG. After the prognosis analysis, we further screened molecules and constructed the prognosis-related ceRNA network. Moreover, the interactions between IncRNA, miRNA, and mRNA were validated by biological experiments.

Results: 854 DELs, 150 DEMs, and 2211 DEGs between metastasis and non-metastasis LUAD patients were identified. Functional enrichment analysis suggested that DEGs were closely related to key biological processes involved in LUAD progression. The prognosis-related ceRNA network included 1 miRNA, 2 IncRNAs, and 4 mRNAs. In this network, MIR155HG and ADAMTS9-AS2 can function as ceRNAs of miR-212 to regulate EPM2AIP1, LAX1, PRICKLE2, and CD226. Moreover, our study confirmed that MIR155HG inhibited the proliferation, migration, and invasion of LUAD cells by sponging miR-212-3p to regulate CD226.

Conclusions: This ceRNA network contributes to understanding the pathogenesis of LUAD. Furthermore, the molecules in the network are valuable predictive factors for LUAD prognosis as well as potential therapeutic biomarkers.

Key words: lung adenocarcinoma, ceRNA network, MIR155HG, miR-212-3p, CD226.

\section{Introduction}

Lung cancer, the most lethal cancer, consists of non-small cell lung cancer (NSCLC) and small cell lung cancer (SCLC) [1-3]. Lung adenocarcinoma (LUAD) is categorised as the most common subtype of NSCLC, accounting for approximately $40 \%$ of all lung cancers [4]. Younger people

\author{
Corresponding author: \\ Dr. Jie Li \\ Department \\ of Thoracic Surgery \\ Beijing Friendship \\ Hospital \\ Capital Medical \\ University \\ 95 Yong'an Road \\ Xicheng District \\ Beijing 100050, China \\ Phone: +86-10-63016616 \\ Fax: +86-10-63016616 \\ E-mail: lijie95cmu@163.com
}


(under 46 years old) and women are more likely to have LUAD than other lung cancers [5]. Smoking is a major cause for most lung cancers; however, LUAD is frequently found in people who have never smoked [6]. The symptoms of LUAD that are more generalised such as subtle shortness of breath and fatigue in the early stages may be ignored or considered to have other causes [7]. Thus, LUAD is usually diagnosed at the advanced stage, and therapeutic strategies are only palliative [8]. The prognosis of LUAD is very poor [9]. In particular, over $50 \%$ of LUAD cases are found to be advanced metastatic disease with a 5 -year survival rate of $4 \%$ [10]. Therefore, studying the molecular mechanism of the pathogenesis of LUAD is necessary to find potential diagnostic approaches and novel treatments.

The occurrence and development of cancer are closely associated with abnormal expression of both non-coding and protein-coding RNAs [11, 12]. In recent years, competitive endogenous RNA (ceRNA) hypothesis has been identified as a novel and important regulatory mechanism [13]. Long non-coding RNAs (IncRNAs) can function as ceRNAs to regulate mRNA expression by competing for shared miRNAs [14]. LncRNAs have received much attention because of their critical roles in various cellular processes [15]. For instance, IncRNA RHPN1-AS1 (RHPN1 antisense RNA 1) can

Table I. Clinical characteristics of lung adenocarcinoma patients

\begin{tabular}{|c|c|c|}
\hline Parameter & $\begin{array}{c}\text { Non-metastasis } \\
\text { group }\end{array}$ & $\begin{array}{c}\text { Metastasis } \\
\text { group }\end{array}$ \\
\hline Median age & 65.8 & 65 \\
\hline \multicolumn{3}{|l|}{ Gender: } \\
\hline Female & 118 & 94 \\
\hline Male & 104 & 95 \\
\hline \multicolumn{3}{|l|}{ M stage: } \\
\hline MO & 222 & 127 \\
\hline M1 & 0 & 25 \\
\hline \multicolumn{3}{|l|}{ N stage: } \\
\hline NO & 222 & 11 \\
\hline N1 & 0 & 175 \\
\hline \multicolumn{3}{|l|}{ T stage: } \\
\hline T1 & 85 & 36 \\
\hline $\mathrm{T} 2$ & 115 & 121 \\
\hline T3 & 17 & 17 \\
\hline $\mathrm{T} 4$ & 5 & 13 \\
\hline \multicolumn{3}{|c|}{ Clinical stage: } \\
\hline 1 & 188 & 0 \\
\hline II & 25 & 82 \\
\hline III & 5 & 80 \\
\hline IV & 0 & 25 \\
\hline
\end{tabular}

sponge miR-596 to regulate LETM1 (leucine zipper and EF-hand containing transmembrane protein 1) expression, thereby promoting the proliferation of ovarian cancer cells [16]. Furthermore, IncRNA HMMR-AS1 (HMMR antisense RNA 1) promotes the growth and metastasis of LUAD by sponging miR-318 to regulate sirt6 expression [17]. To better understand the roles of IncRNAs in cancer, many IncRNA-miRNA-mRNA networks have been constructed based on the ceRNA hypothesis [18] Li et al. analysed the IncRNA profiles from 570 LUAD cases in the TCGA database and established 3 ceRNA (IncRNA-miRNA-mRNA) networks [19]. Although some reports have constructed ceRNA networks using different bioinformatics methods to understand the pathogenesis of LUAD, studies of IncRNA-miRNA-mRNA networks related to prognosis are few in LUAD.

The present study identified differentially expressed IncRNAs (DELs), differentially expressed miRNAs (DEMs), and differentially expressed genes (DEGs) between metastasis and non-metastasis LUAD patients from the TCGA database. Based on these molecules, we constructed IncRNA-miRNAmRNA networks and validated a IncRNA-miRNAmRNA network using biological experiments. Our study contributes to understanding the molecular pathogenesis of LUAD and finding appropriate, targeted therapeutic biomarkers.

\section{Material and methods}

\section{Data sources}

LUAD RNA sequencing (RNA-Seq) data, miRNASeq data, and corresponding clinical information were obtained from The Cancer Genome Atlas (TCGA) database (https://www.cancer.gov/aboutnci/organization/ccg/research/structural-genomics/tcga). Based on the patients' clinical information, they were divided into metastasis and non-metastasis groups: patients at the NO and MO stages were classified as the non-metastasis group; patients at the N1-N3 or M1 stage were classified as the metastasis group; and patients with no information of the $N$ and $M$ stages were excluded. Thus, the non-metastasis group contained 222 cases, and the metastasis group included 189 cases. We used transcriptome annotation files from the GENCODE database to identify mRNAs and IncRNAs and excluded genes that had a mean expression of less than 0.1 . The clinical characteristics of LUAD patients are listed in Table I.

\section{Data preprocessing and differential expression analysis}

Data in the raw count format from TCGA were normalised with the transcripts per million (TPM) method and underwent a log2 transformation 
using the DESeq2 package in $\mathrm{R}$ language (version 3.4.1; https://www.r-project.org/). The differentially expressed IncRNAs (DELs), miRNAs (DEMs), and genes (DEGs) between metastasis and non-metastasis LUAD patients were identified using the Linear Models for Microarray Data (Limma, version 3.34.0; http://www.bioconductor.org/packages/release/bioc/html/limma.html) package in R. A $p$-value $<0.01$ was designated as the threshold. The information of all obtained DELs, DEMs, and DEGs is listed in Supplementary Table SI.

\section{ceRNA network construction}

Firstly, miRcode (http://www.mircode.org/) was utilised to find potential IncRNAs that sponge the DEMs. LncRNAs that met the following two criteria were retained: overlapped with the DELs and a negative correlation with DEMs. Secondly, the target genes of DEMs were identified using miRTarBase (http://mirtarbase.mbc.nctu.edu.tw/php/index. php), miRDB (http://mirdb.org/), and TargetScan (http://www.targetscan.org/vert_71/). Target genes that met the following 3 criteria were retained: predicted by at least 2 websites, overlapped with the DEGs, and a negative correlation with DEMs. Finally, we integrated the interactions of IncRNA-miRNA and miRNA-mRNA to construct the ceRNA network using Cytoscape software (version 3.4).

\section{Functional enrichment analysis}

To study the function of mRNAs in the ceRNA network, we used the clusterProfiler package to perform GO (Gene Ontology) and KEGG (Kyoto encyclopaedia of genes and genomes) pathway enrichment analyses. GO terms contain BP (biological process), MF (molecular function), and CC (cellular component) categories. A $p$-value $<0.05$ was designated as the cut-off criterion.

\section{Prognosis-related ceRNA network}

To further screen the ceRNA networks, we downloaded the expression data and clinical information of IncRNAs, miRNAs, and mRNAs in the ceRNA network from the TCGA database and performed a univariate cox regression analysis to identify prognosis-related IncRNAs, miRNAs, and mRNAs using the survival package (version 2.43-3). The median expression level of each IncRNA, miRNA, and mRNA in the ceRNA network was set as the cut-off value and the samples were divided into a low-expression group and a high-expression group. The Kaplan-Meier method was used to draw overall survival (OS) curves, and the logrank test was used to assess the difference of OS between the low-expression and high-expression groups. A $p$-value of $<0.05$ was considered statistically significant. The prognosis-related IncRNAs,
miRNAs, and mRNAs were used to construct the prognosis-related ceRNA network using Cytoscape software (version 3.4).

\section{Cell culture and transfection}

Human normal lung epithelial cells (BEAS-2B) and LUAD cell lines (NCl-H1299, A549, NCl-H1975) were purchased from the Cell Bank of the Chinese Academy of Sciences (Shanghai, China). LUAD cell lines were incubated in RPMI-1640 medium (Gibco) supplemented with $10 \%$ foetal bovine serum (Gibco) and 1\% penicillin/streptomycin (Gibco) and maintained in a humidified incubator with $5 \% \mathrm{CO}_{2}$ at $37^{\circ} \mathrm{C}$. BEAS-2B cells were cultured using a BEGM kit (Lonza, Basel, Switzerland) and maintained in a humidified incubator with $5 \% \mathrm{CO}_{2}$ at $37^{\circ} \mathrm{C}$.

The MIR155HG plasmid and scramble plasmid were synthesised from FITGENE (Guangzhou, China). The siRNA MIR155HG (si-MIR155HG) and siRNA-negative control (si-NC) were purchased from Invitrogen. These vectors were transfected into LUAD cells using Lipofectamine 3000 transfection reagent (Invitrogen, Carlsbad, CA, USA).

\section{Real-time quantitative PCR (RT-qPCR)}

Total RNA from LUAD cells was extracted with TRIzol reagent (Invitrogen). The synthesis of cDNA was performed using the QuantiTect Reverse Transcription Kit (Qiagen, USA). Next, RT-qPCR was carried out by the SYBRTM Green PCR Kit (Life Technologies) under Applied Biosystems ViiAt 7 Real-Time PCR System (Life Technologies). The relative expression of target genes was assessed using the $2^{-\Delta \Delta c t}$ method with $\beta$-actin or $U 6$ as internal controls. Table II shows the primers used for RT-qPCR.

\section{Cell counting kit 8 (CCK-8) assay}

At $48 \mathrm{~h}$ post-transfection, cells were harvested and seeded in 96-well plates at a density of $1 \times 10^{4}$ cells per well. The CCK-8 reagent $(10 \mu \mathrm{l}$, Engreen Biosystem) was added to cells after 24, 48, 72, and

Table II. RT-qPCR primer sequences

\begin{tabular}{|lc|}
\hline Primers & Sequence $\left(\mathbf{5}^{\prime} \rightarrow \mathbf{3}^{\prime}\right.$ ) \\
\hline MIR155HG forward & GAGTGCTGAAGGCTTGCTGT \\
\hline MIR155HG reverse & TTGAACATCCCAGTGACCAG \\
\hline miR-212-3p forward & GGTAACAGTCTCCAGTCA \\
\hline miR-212-3p reverse & GCAATTGCACTGGATACG \\
\hline CD226 forward & CAGCACTCACATCTCAAGAACC \\
\hline CD226 reverse & TGTTCTCGGCAAAGGGAACT \\
\hline$\beta$-actin forward & TCACCCACACTGTGCCCATCTACGA \\
\hline$\beta$-actin reverse & CAGCGGAACCGCTCATTGCCAATGG \\
\hline U6 forward & GCTTCGGCAGCACATATACTAAAAT \\
\hline U6 reverse & CGCTTCACGAATTTGCGTGTCAT \\
\hline
\end{tabular}


$96 \mathrm{~h}$ of incubation. Next, the cells were cultured for another $4 \mathrm{~h}$ and the optical density was measured using a microplate reader at $450 \mathrm{~nm}$.

\section{Wound healing assay}

At $48 \mathrm{~h}$ post-transfection, cells were incubated to $90 \%$ confluence using RPMI-1640 medium supplemented with $10 \%$ foetal bovine serum and $1 \%$ penicillin/streptomycin in 6-well plates. A sterile $100 \mu \mathrm{l}$ pipette tip was used to make straight lines through the cell monolayer. Next, the cells were washed with phosphate-buffered saline (PBS) and cultured with serum-free RPMI-1640 medium for 24 h. An Olympus BX51 light microscope (Olympus Corporation) was used to image the cells.

\section{Transwell invasion assay}

The transfected cells were collected, suspended in serum-free RPMI-1640 medium, and seeded in the upper Transwell chamber of 24-well plates at a density of $1 \times 10^{5}$ cells per chamber. The lower chamber was filled with RPMI-1640 medium and $10 \%$ FBS as a chemoattractant. After incubation for $24 \mathrm{~h}$, the invaded cells were fixed using 4\% paraformaldehyde for $30 \mathrm{~min}$ at room temperature and stained in $0.1 \%$ crystal violet for $20 \mathrm{~min}$. After washing with PBS, the cells were imaged using an Olympus BX51 light microscope (Olympus Corporation; magnification 200x).

\section{Dual luciferase reporter assay}

The putative binding sites of miR-212-3p with MIR155HG and CD226 were identified using miRcode (http://www.mircode.org/) and miRTarBase (http://mirtarbase.mbc.nctu.edu.tw/php/index. php). The 3'-untranslated region (UTR) fragments of MIR155HG and CD226 containing miR-212-3p wild-type (WT) or mutant (MUT) binding sites were amplified using PCR and cloned into PMIR-REPOR ${ }^{\text {TM }}$ Luciferase vectors (Promega) based on standard protocols to form MIR155HG-WT, MIR155HG-MUT, CD226-WT, and CD226-MUT luciferase reporter vectors. These reporter vectors with miR-212-3p mimic or miRNA control were co-transfected into LUAD cells using Lipofectamine 3000 reagent (Invitrogen) and cells were incubated for $48 \mathrm{~h}$ at $37^{\circ} \mathrm{C}$. After transfection, the cells were collected using a lysis buffer, and luminescence was measured according to the protocol of the dual luciferase reporter assay kit (Dual-Luciferase ${ }^{\circledR} 1000$ Assay kit, Promega). The firefly luciferase activity was normalised to Renilla luciferase activity.

\section{Western blot}

Total protein was lysed from LUAD cells with RIPA lysis buffer (Beyotime Institute of Biotechnol- ogy) on ice. Protein concentration was measured using a BCA Protein Assay Kit (ThermoFisher Scientific, USA). Proteins $(20 \mu \mathrm{g})$ were equally separated on a $10 \%$ SDS-PAGE gel and electro-transferred onto PVDF membranes. Membranes, after being blocked using 5\% non-fat milk for $1 \mathrm{~h}$ at room temperature, were incubated with primary antibodies against CD226 (Cat. No. \#66631, dilution $1: 1000$ ) and $\beta$-actin (Cat. No. \#4970, dilution 1 : 1000) at 4 overnight. The membranes were washed using TBS buffer with $0.05 \%$ Tween-20 and incubated with a secondary antibody (antirabbit IgG, HRP-linked antibody, Cat. No. \#7074, dilution 1 : 1000) for $1 \mathrm{~h}$ at room temperature. The primary and secondary antibodies were purchased from Cell Signaling Technology. A chemiluminescence detection kit (Aidlab Biotechnology, Beijing, China) was utilised to perform the colour rendering of protein bands. The protein density was calculated by ImageJ software.

\section{Statistical analysis}

All experiments in the present study were carried out in triplicate. The data are presented as the mean \pm standard deviation (SD). GraphPad 7 software was used to perform statistical analysis. Student's $t$-test was applied to test for differences between two groups, and a one-way ANOVA with Tukey's test evaluated the differences among multiple groups. A $p$-value of $<0.05$ was considered significant.

\section{Results}

\section{Differential expression analysis}

After LUAD datasets were preprocessed by the DESeq2 package, 854 DELs (785 downregulated and 69 upregulated), 150 DEMs (64 downregulated and 86 upregulated), and 2211 DEGs (1246 downregulated and 965 upregulated) between metastasis and non-metastasis LUAD patients were obtained using the Limma package (Supplementary Table SI). The expression levels of the top 20 DELs, DEMs, and DEGs are shown in a heatmap (Figure 1).

\section{ceRNA network construction}

Interaction of IncRNA with miRNA was predicted by miRcode, and the interaction between miRNA and mRNA was identified using miRTarBase, miRDB, and TargetScan. Furthermore, the data of DELs, DEMs, and DEGs were combined with the interactions among InCRNA, miRNA, and mRNA to obtain 44 IncRNA-miRNA interaction pairs and 132 miRNA-mRNA interaction pairs, including 15 IncRNAs (e.g. MIR155HG, LINC00324, LINC00337), 7 miRNAs (e.g. hsa-mir-200a, hsa- 
LncRNA MIR155HG functions as a ceRNA for inhibition of lung adenocarcinoma growth and prediction of prognosis

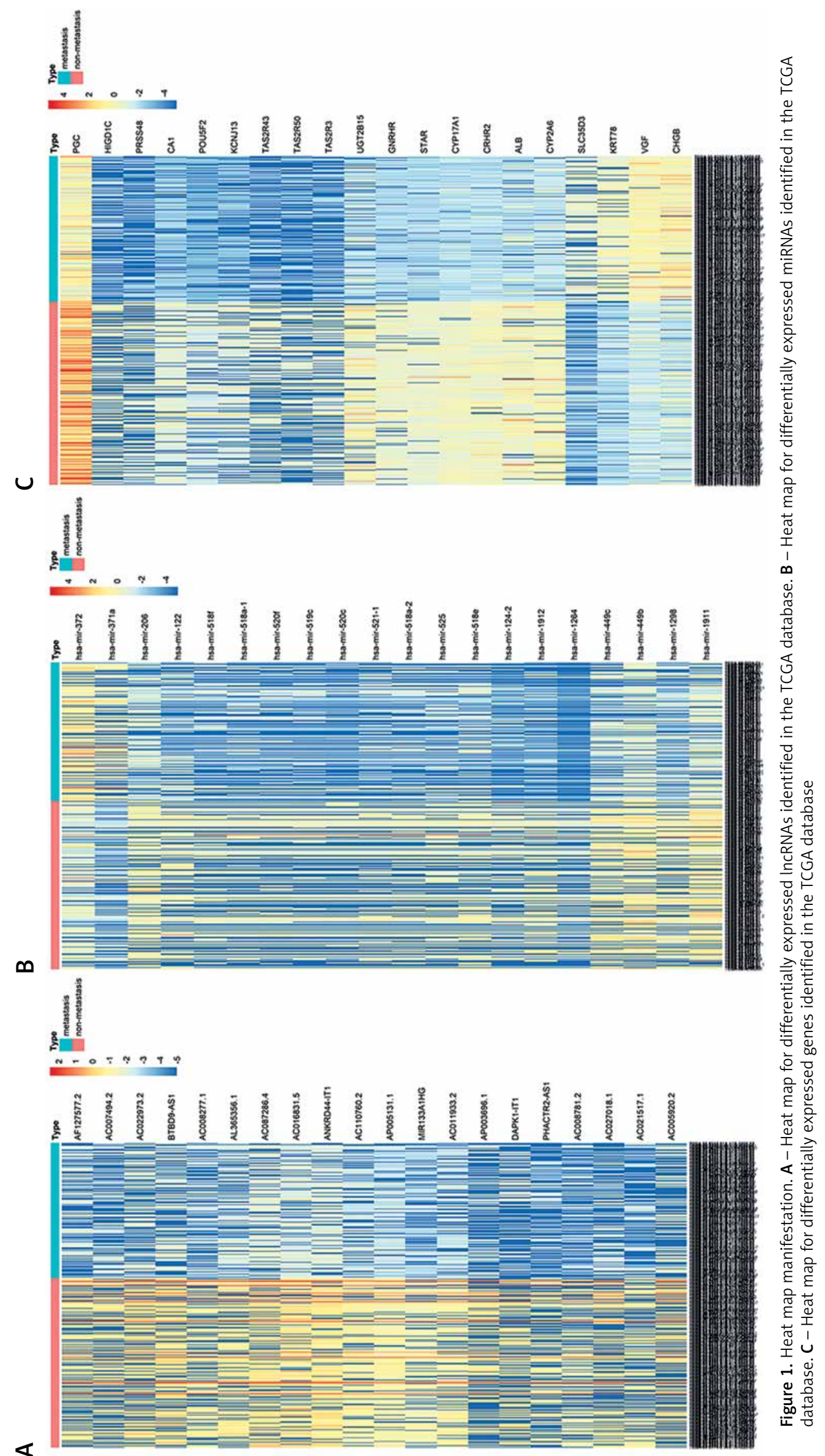




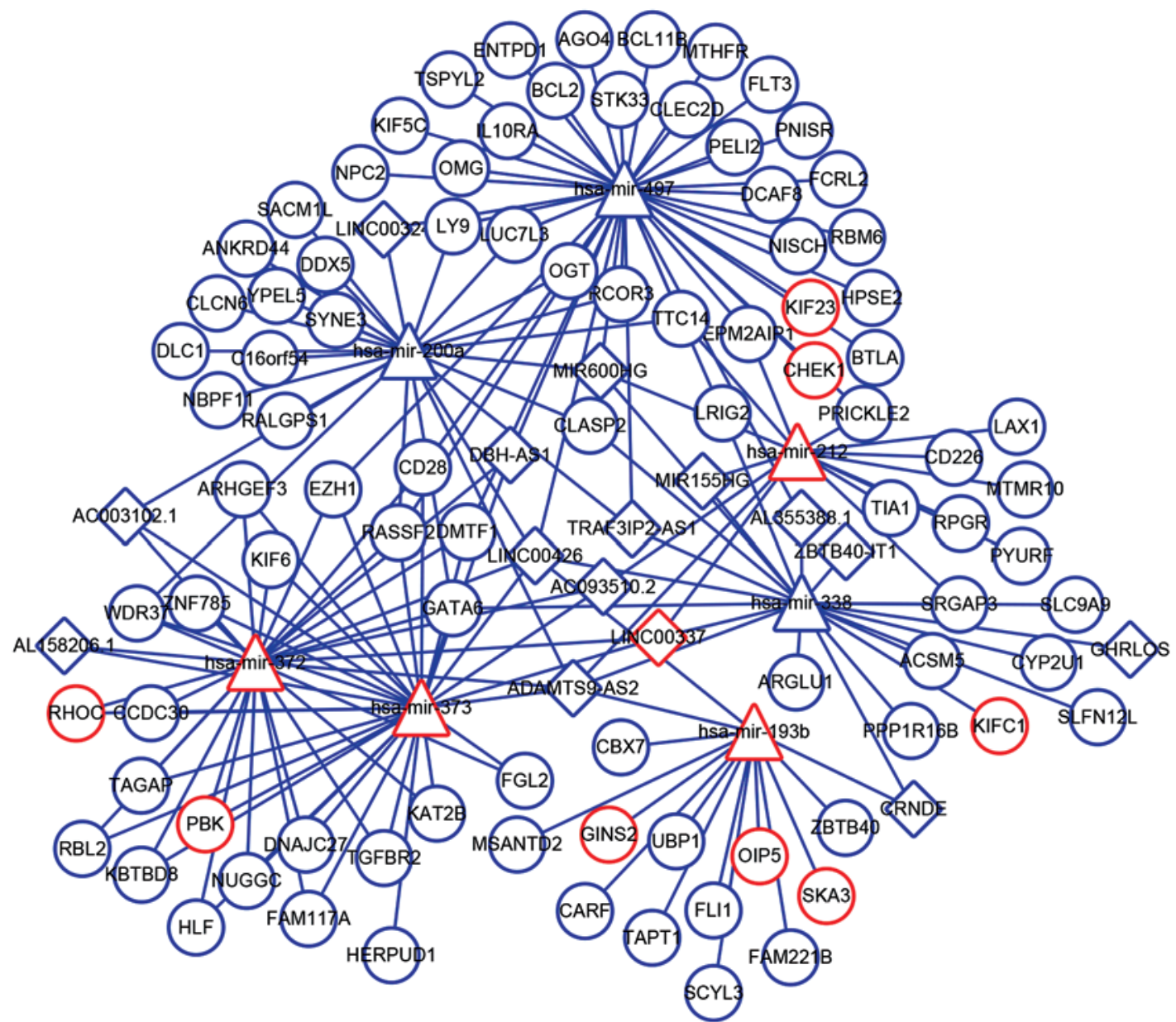

Figure 2. Competing endogenous RNAs (ceRNAs) interaction network of IncRNA-miRNA-mRNA. Square nodes represent IncRNAs, triangle nodes represent miRNAs, and round nodes represent mRNAs. Edges represent the possible associations between IncRNAs, miRNAs, and mRNAs. Red, upregulated; Blue, downregulated

mir-212, hsa-mir-193b), and 91 mRNAs (e.g. RHOC, CBX7, CD226). Then, the ceRNA network was constructed by Cytoscape software (Figure 2), which indicated the potential interaction relationships among IncRNA, miRNA, and mRNA.

\section{Functional enrichment analysis}

mRNAs in the ceRNA network were analysed using the clusterProfiler package to find their underlying functions in LUAD. The results of the GO analysis are shown in Figure $3 \mathrm{~A}$. For BP, mRNAs in the ceRNA network were primarily enriched in small GTPase mediated signal transduction. For CC, mRNAs were mainly enriched in chromosomal region. For MF, mRNAs were chiefly enriched in chromatin binding. In addition, mRNAs in the ceRNA network were assessed by KEGG pathway analysis. The top 10 pathways significantly correlated with LUAD metastasis are listed in Figure 3 B. For example, transcriptional misregulation in cancer and the p53 signalling pathway are closely associated with the progression of LUAD.

\section{Prognosis-related ceRNA network}

To further screen the ceRNA networks, we downloaded the corresponding overall survival information in the ceRNA network from TCGA and performed a univariate cox regression analysis using the survival package. The results found, prognosis-related, 4 IncRNAs, 1 miRNA, and 49 mRNAs (Supplementary Table SII). Then, the data were combined with the interactions among IncRNA, miRNA, and mRNA to obtain 2 IncRNA-miRNA interaction pairs and 4 miRNA-mRNA interaction pairs, which consisted of 1 miRNA, 2 IncRNAs, and 4 mRNAs. OS curves of these molecules were drawn using Kaplan-Meier method with log-rank test (Figure $4 \mathrm{~A}$ ). For instance, the high expression of MIR155HG had higher OS values, indicating better prognosis, as compared to low expression of MIR155HG. The high expression of miR-212 showed poor prognosis compared with the low expression of miR-212. As shown in Figure $4 \mathrm{~B}$, the prognosis-related ceRNA network was established using Cytoscape software. In this network, 
A

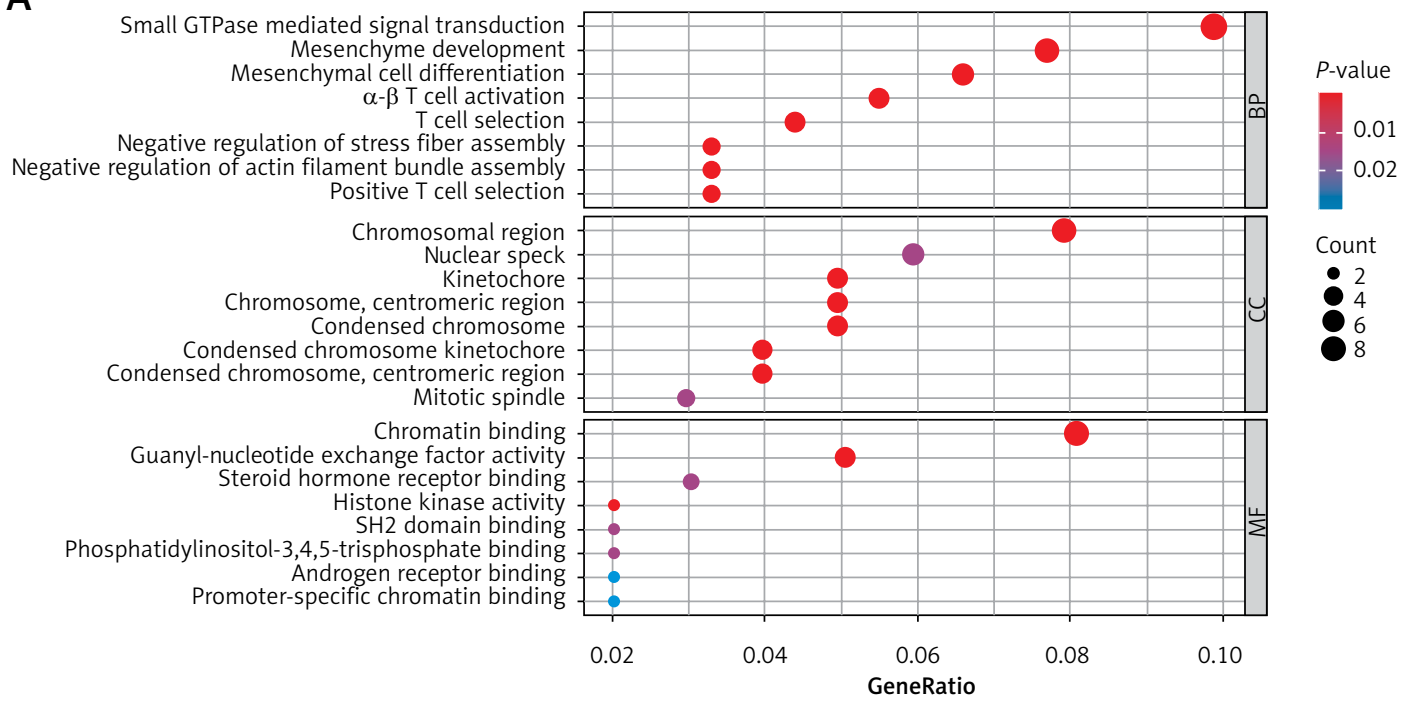

B

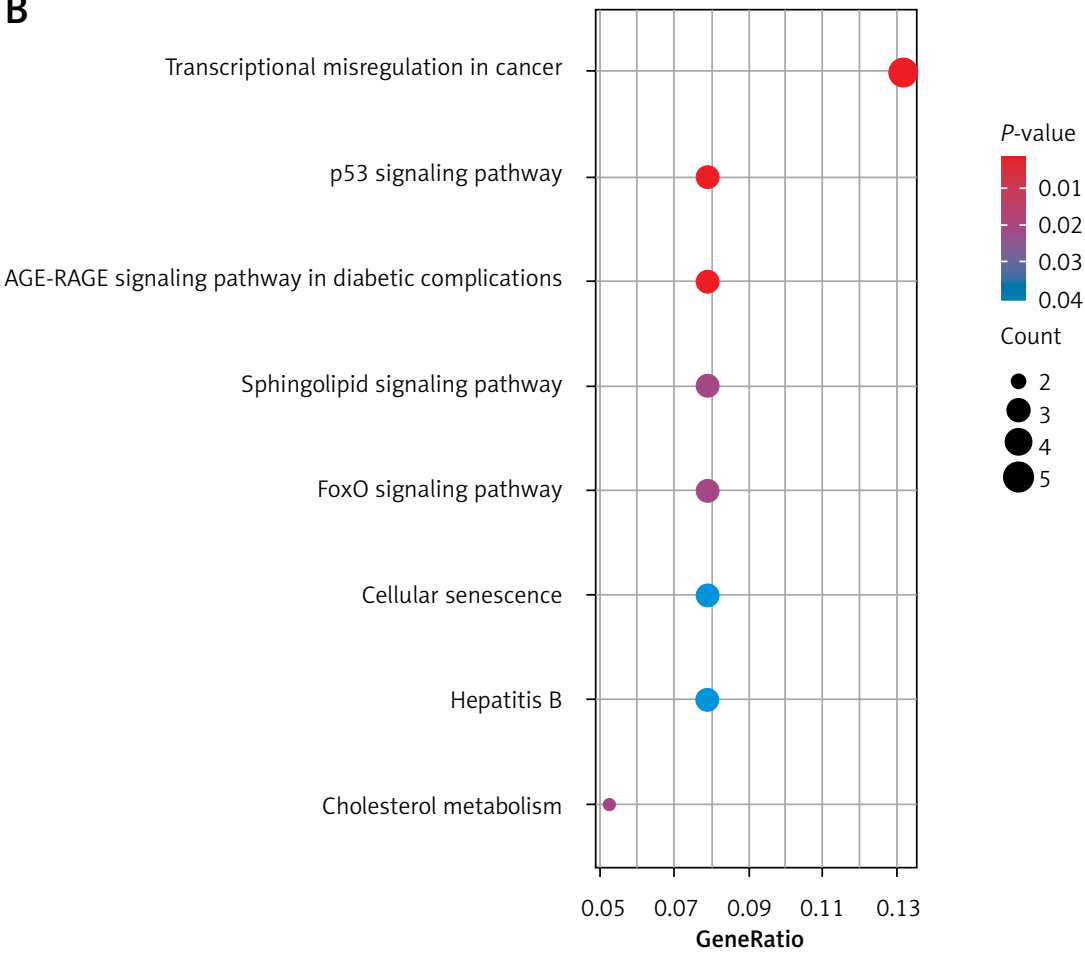

Figure 3. Functional enrichment analysis. GO (A) and KEGG (B) functional enrichment analysis for the genes in the ceRNA network

MIR155HG and ADAMTS9-AS2 can function as CeRNAs of miR-212 to regulate EPM2AIP1, LAX1, PRICKLE2, and CD226.

Additionally, the correlation between clinical characteristics and molecule expression in the prognosis-related ceRNA network was assessed by $\chi^{2}$ test and one-way ANOVA. As shown in Table III, statistical analysis indicated that the expression of six molecules (MIR155HG, ADAMTS9AS2, EPM2AIP1, PRICKLE2, CD226, and mir-212) was significantly associated with the degree of spread to regional lymph nodes. The expression of ADAMTS9-AS2 and PRICKLE2 exhibited an obvious correlation with gender. There was a correlation of PRICKLE2 expression with distant metastasis. Also, the expression levels of LAX1 were correlated with the size of the original (primary) tumour. Additionally, MIR155HG, LAX1, CD226, and mir-212 expression levels were associated with clinical stage.

To validate the roles of the prognosis-related ceRNA network in LUAD, we selected MIR155HG, miR-212, and CD226 from this network according to the previous reports regarding the interaction between the molecules in the ceRNA network and $\operatorname{LUAD}[20,21]$ and performed in vitro experiments to explore their effects on LUAD cells. 

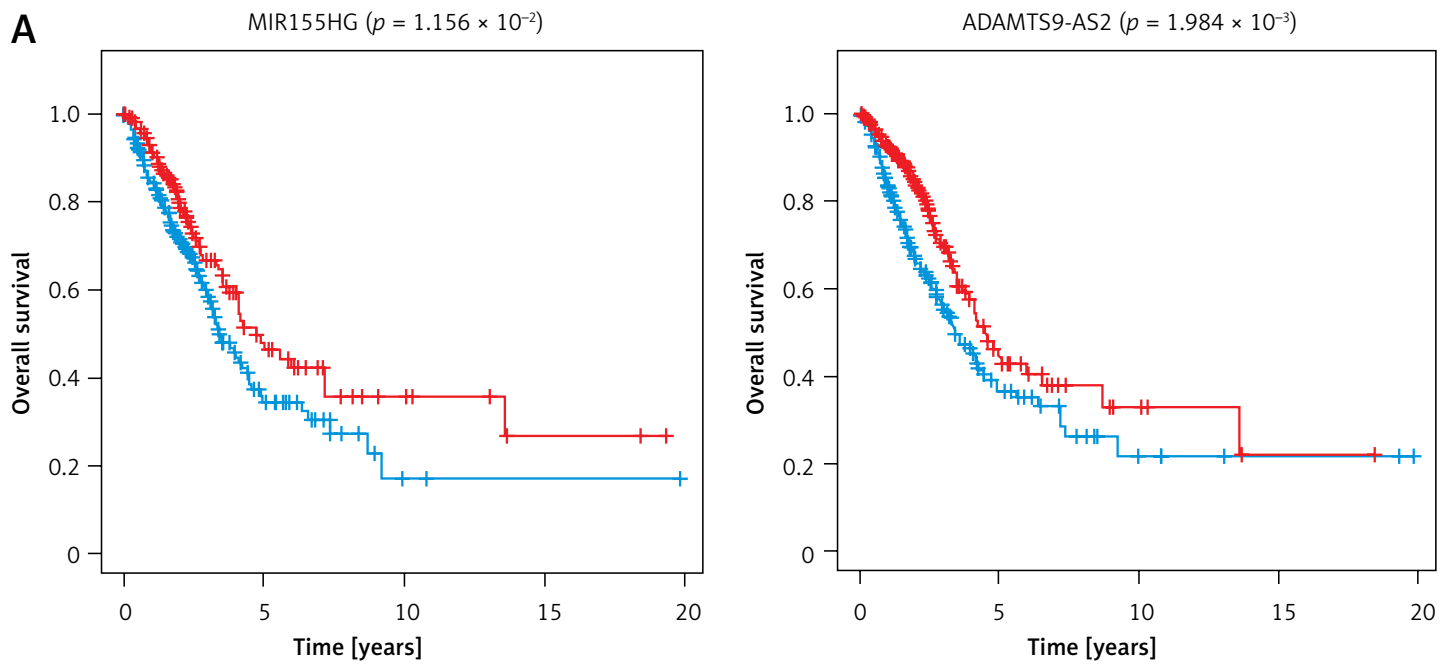

- Low expression - High expression

— Low expression - High expression
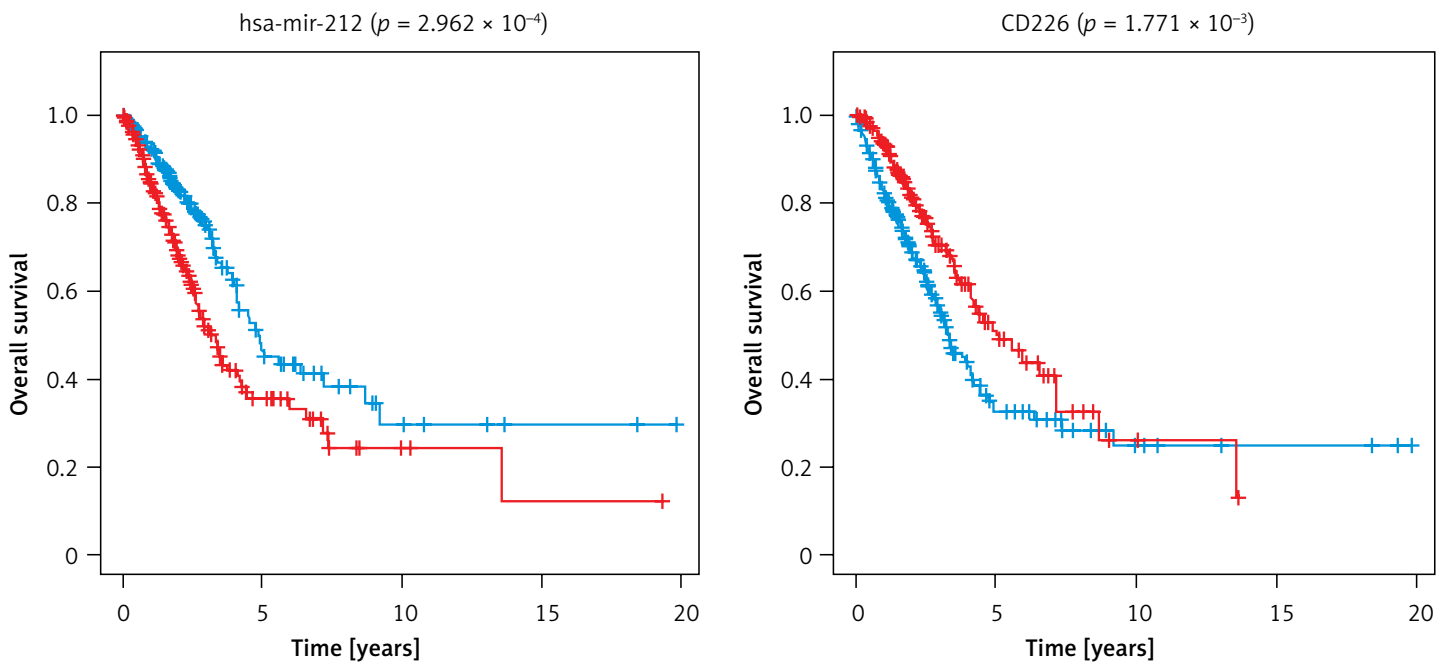

- Low expression - High expression

EPM2AIP1 $\left(p=4.912 \times 10^{-2}\right)$

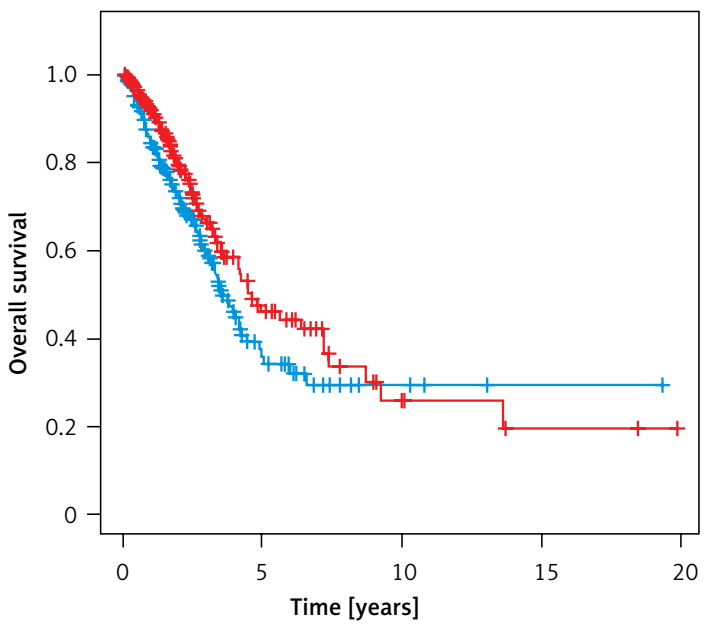

— Low expression — High expression

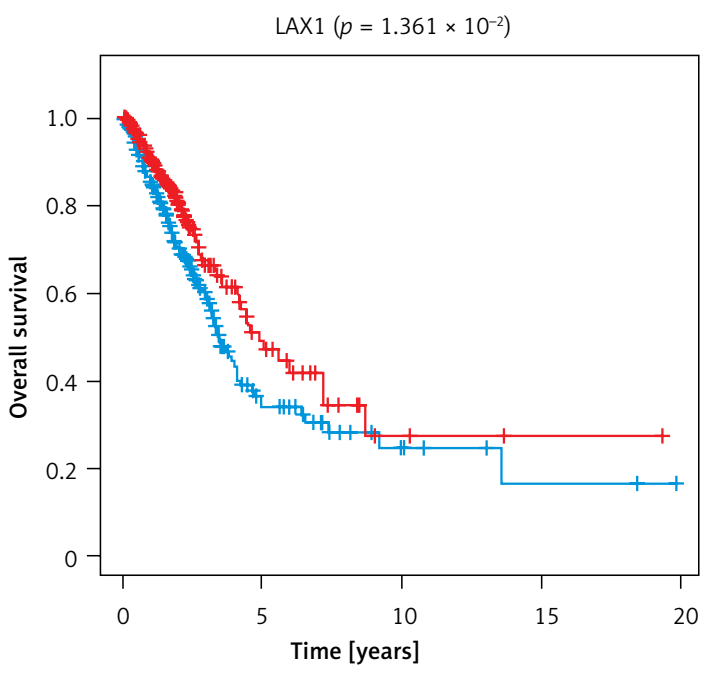

- Low expression - High expression

- Low expression $\quad$ - High expression

Figure 4. Prognosis-related ceRNA network. A - Overall survival curves of the IncRNA, miRNA, and mRNA of crucial ceRNA axis in which all IncRNAs, miRNAs, and mRNAs were related to prognosis 
A

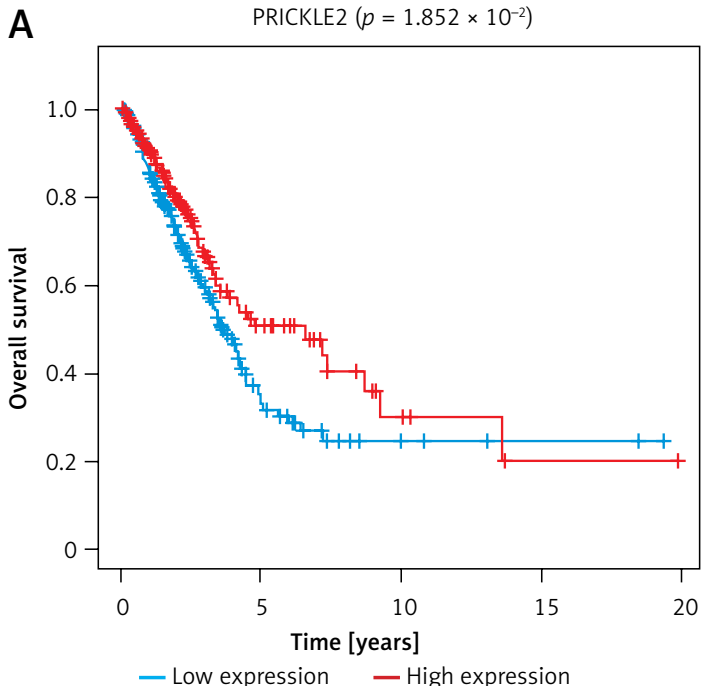

B

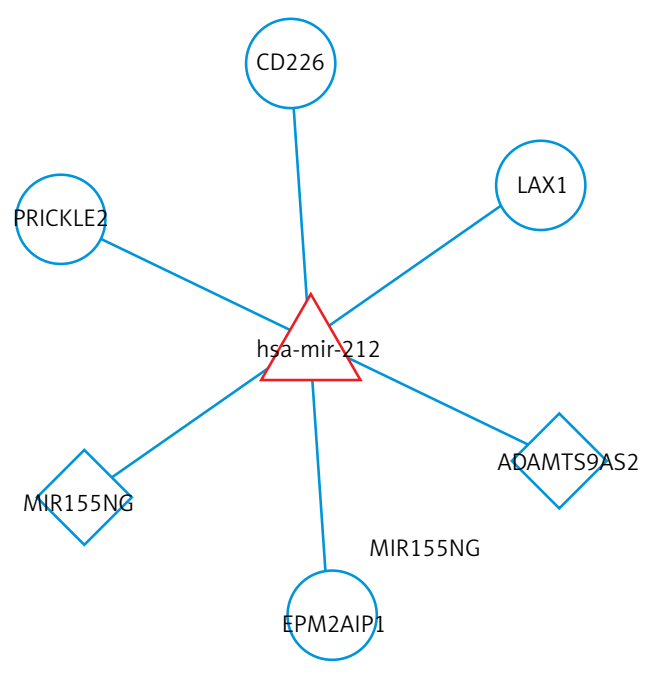

Figure 4. Cont. A - Overall survival curves of the IncRNA, miRNA, and mRNA of crucial ceRNA axis in which all IncRNAs, miRNAs, and mRNAs were related to prognosis. B - The image of the prognosis-related ceRNA network; Square nodes represent IncRNAs; triangle nodes represent miRNAs; round nodes represent mRNAs. Edges represent the possible associations between IncRNAs, miRNAs, and mRNAs. Red, upregulated; Blue, downregulated

LncRNA MIR155HG inhibits the proliferation, migration, and invasion of LUAD cells

Firstly, RT-qPCR observed that the expression levels of MIR155HG in LUAD cell lines (NCI-H1299, A549, $\mathrm{NCl}-\mathrm{H} 1975)$ were lower than in human normal lung epithelial cells (BEAS-2B), which was consistent with the bioinformatics analysis results (Figure 5 A). Additionally, A549 and NCl-H1975 cells were chosen for the following experiments, because they had the lowest relative expression. Also, the transfection efficiency of MIR155HG plasmid in $\mathrm{A} 549$ and $\mathrm{NCl}-\mathrm{H} 1975$ cells had a relatively high level compared with scramble plasmid as the control group (Figure 5 B). Furthermore, CCK8 assay results showed that MIR155HG overexpression obviously inhibited the proliferation of A549 and $\mathrm{NCl}-\mathrm{H} 1975$ cells when compared with the control group (Figure 5 C). Moreover, compared with the control group, MIR155HG overexpression significantly suppressed the migration and invasion of $\mathrm{A} 549$ and $\mathrm{NCl}-\mathrm{H} 1975$ cells (Figures $5 \mathrm{D}-\mathrm{G}$ ).

\section{MIR155HG functions as a ceRNA of}

miR-212-3p and CD226 is a direct target of miR-212-3p

To verify the interaction among MIR155HG, miR-212-3p, and CD226, we separately performed dual luciferase reporter assay, RT-qPCR, and western blot. The binding sites of MIR155HG with miR212-3p were identified by the miRcode database (Figure $6 \mathrm{~A}$ ). The co-transfection of MIR155HG-WT with miR-212-3p mimic in LUAD cells considerably down-regulated the relative luciferase activity in comparison to its co-transfection with miRNA mimic control (NC mimic); however, there was no significant difference between MIR155HG-MUT co-transfected with miR-212-3p mimic and the NC mimic (Figure 6 B). Dual luciferase reporter assay confirmed that MIR155HG could directly interact with miR-212-3p (Figure 6 B). After LUAD cells were respectively transfected with MIR155HG plasmid, scramble plasmid, siRNA MIR155HG (si-MIR155HG), and siRNA control (si-NC), the expression of miR-212-3p was up-regulated by MIR155HG knockdown, while the level of miR-212$3 p$ was down-regulated by MIR155HG overexpression (Figure $6 \mathrm{C}$ ). MIR155HG negatively regulated miR-212-3p expression. Collectively, MIR155HG could function as a ceRNA of miR-212-3p.

The binding sites between miR-212-3p and CD226 were predicted by the miRTarBase database (Figure $6 \mathrm{D}$ ). Dual luciferase reporter assay further confirmed that miR-212-3p could directly target CD226 (Figure 6 E). RT-qPCR and western blot showed that miR-212-3p mimic downregulated the expression of CD226, but miR-212-3p inhibitor up-regulated CD226 expression (Figures 6 $\mathrm{F}-\mathrm{H}$ ). Therefore, CD226 was a target gene of miR212-3p. These findings confirmed the results of the prognosis-related ceRNA network.

\section{MIR155HG inhibits the proliferation, migration, and invasion of LUAD cells through regulation of the miR-212-3p/ CD226 axis}

To study the roles of MIR155HG, miR-212$3 p$, and CD226 in LUAD cells, LUAD cells after co-transfection of MIR155HG plasmid combined with or without miR-212-3p mimic were respectively determined by western blot, CCK8, wound 
Jing Wang, Jie Li

Table III. The correlation between clinical characteristics and molecules expression in a prognosis-related ceRNA network

\begin{tabular}{|c|c|c|c|c|c|c|c|c|c|c|c|c|}
\hline \multirow[t]{2}{*}{ Characteristics } & \multicolumn{3}{|c|}{ MIR155HG } & \multicolumn{3}{|c|}{ ADAMTS9-AS2 } & \multicolumn{3}{|c|}{ EPM2AIP1 } & \multicolumn{3}{|c|}{ LAX1 } \\
\hline & Low & High & $P$-value & Low & High & $P$-value & Low & High & $P$-value & Low & High & $P$-value \\
\hline Gender: & & & 0.1633 & & & 0.0284 & & & 0.1633 & & & 0.3194 \\
\hline Male & 105 & 90 & & 109 & 86 & & 105 & 90 & & 103 & 92 & \\
\hline Female & 97 & 111 & & 93 & 115 & & 97 & 111 & & 99 & 109 & \\
\hline Tumour (T): & & & 0.1806 & & & 0.7674 & & & 0.4577 & & & 0.0177 \\
\hline $\mathrm{T} 1+\mathrm{T} 2$ & 170 & 179 & & 177 & 172 & & 172 & 177 & & 166 & 183 & \\
\hline $\mathrm{T} 3+\mathrm{T} 4$ & 31 & 21 & & 25 & 27 & & 29 & 23 & & 34 & 18 & \\
\hline $\begin{array}{l}\text { Regional lymph } \\
\text { nodes }(N) \text { : }\end{array}$ & & & 0.0085 & & & 0.0155 & & & 0.0003 & & & 0.0687 \\
\hline NO & 101 & 128 & & 103 & 126 & & 97 & 132 & & 105 & 124 & \\
\hline N1 & 99 & 72 & & 98 & 73 & & 104 & 67 & & 95 & 76 & \\
\hline $\begin{array}{l}\text { Distant metastasis } \\
(\mathrm{M}) \text { : }\end{array}$ & & & 0.6799 & & & 0.8388 & & & 0.3078 & & & 0.1005 \\
\hline MO & 171 & 170 & & 172 & 169 & & 174 & 167 & & 172 & 169 & \\
\hline M1 & 14 & 11 & & 12 & 13 & & 10 & 15 & & 17 & 8 & \\
\hline Clinical stage: & & & 0.0243 & & & 0.1772 & & & 0.4328 & & & 0.0024 \\
\hline$I+I I$ & 134 & 154 & & 138 & 150 & & 140 & 148 & & 130 & 158 & \\
\hline III + IV & 65 & 44 & & 61 & 48 & & 58 & 51 & & 68 & 41 & \\
\hline \multirow[t]{2}{*}{ Characteristics } & \multicolumn{3}{|c|}{ PRICKLE2 } & \multicolumn{3}{|c|}{ CD226 } & \multicolumn{3}{|c|}{ hsa-mir-212 } & & & \\
\hline & Low & High & $P$-value & Low & High & $P$-value & Low & High & $P$-value & & & \\
\hline Gender: & & & 0.0168 & & & 0.073 & & & 0.2736 & & & \\
\hline Male & 110 & 85 & & 107 & 88 & & 93 & 104 & & & & \\
\hline Female & 92 & 116 & & 95 & 113 & & 109 & 97 & & & & \\
\hline Tumour (T): & & & 1 & & & 0.1806 & & & 0.1399 & & & \\
\hline $\mathrm{T} 1+\mathrm{T} 2$ & 175 & 174 & & 170 & 179 & & 180 & 169 & & & & \\
\hline $\mathrm{T} 3+\mathrm{T} 4$ & 26 & 26 & & 31 & 21 & & 21 & 31 & & & & \\
\hline $\begin{array}{l}\text { Regional lymph } \\
\text { nodes }(N) \text { : }\end{array}$ & & & 0.026 & & & 0.0046 & & & 0.0046 & & & \\
\hline NO & 103 & 126 & & 100 & 129 & & 129 & 100 & & & & \\
\hline N1 & 97 & 74 & & 100 & 71 & & 71 & 100 & & & & \\
\hline $\begin{array}{l}\text { Distant metastasis } \\
(M):\end{array}$ & & & 0.0232 & & & 0.2177 & & & 0.8308 & & & \\
\hline MO & 164 & 177 & & 172 & 169 & & 176 & 167 & & & & \\
\hline M1 & 18 & 7 & & 16 & 9 & & 11 & 12 & & & & \\
\hline Clinical stage: & & & 0.2609 & & & 0.0007 & & & 0.0235 & & & \\
\hline$I+I I$ & 139 & 149 & & 129 & 159 & & 156 & 135 & & & & \\
\hline III + IV & 60 & 49 & & 70 & 39 & & 43 & 64 & & & & \\
\hline
\end{tabular}



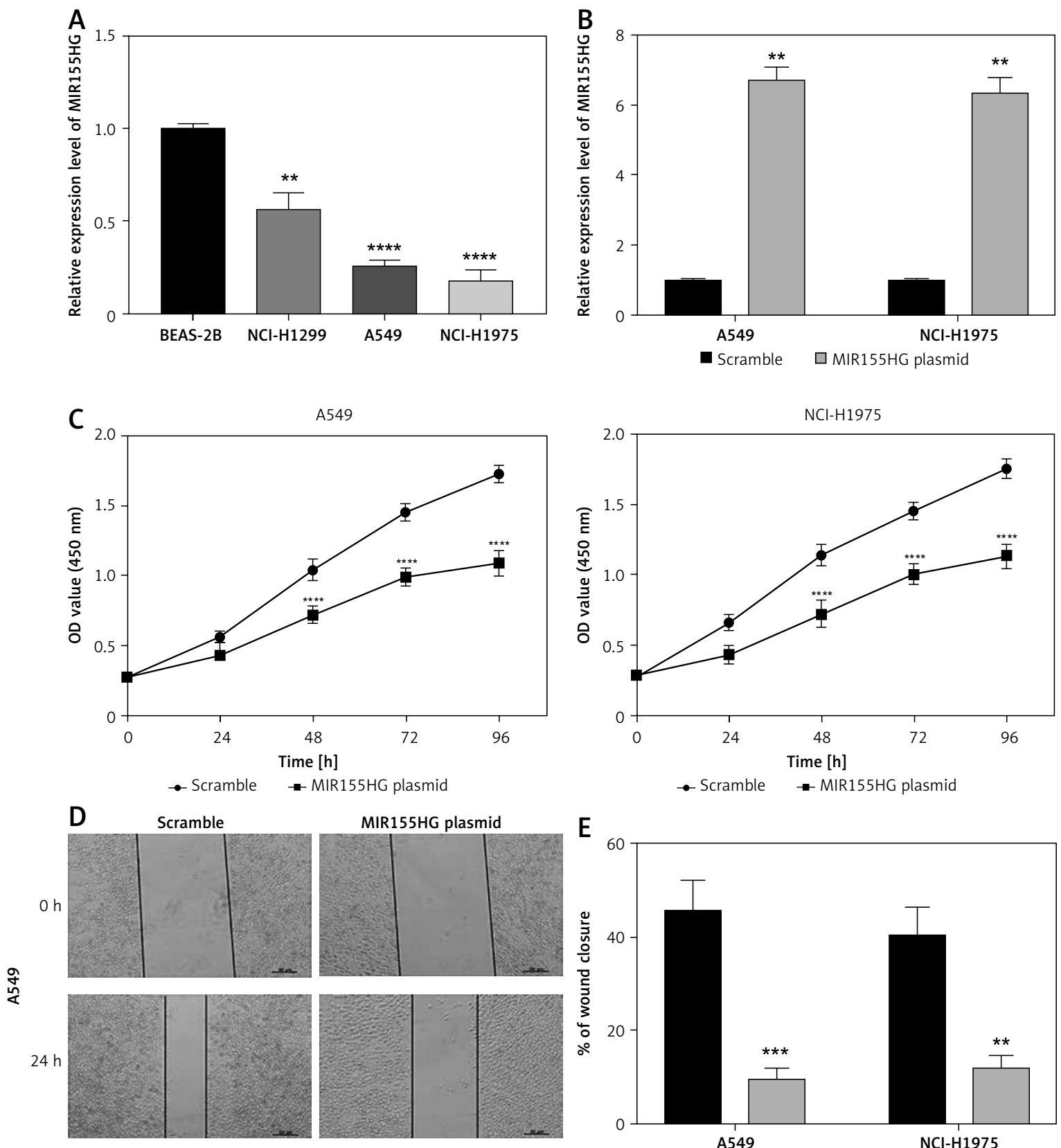

$\mathrm{E}$
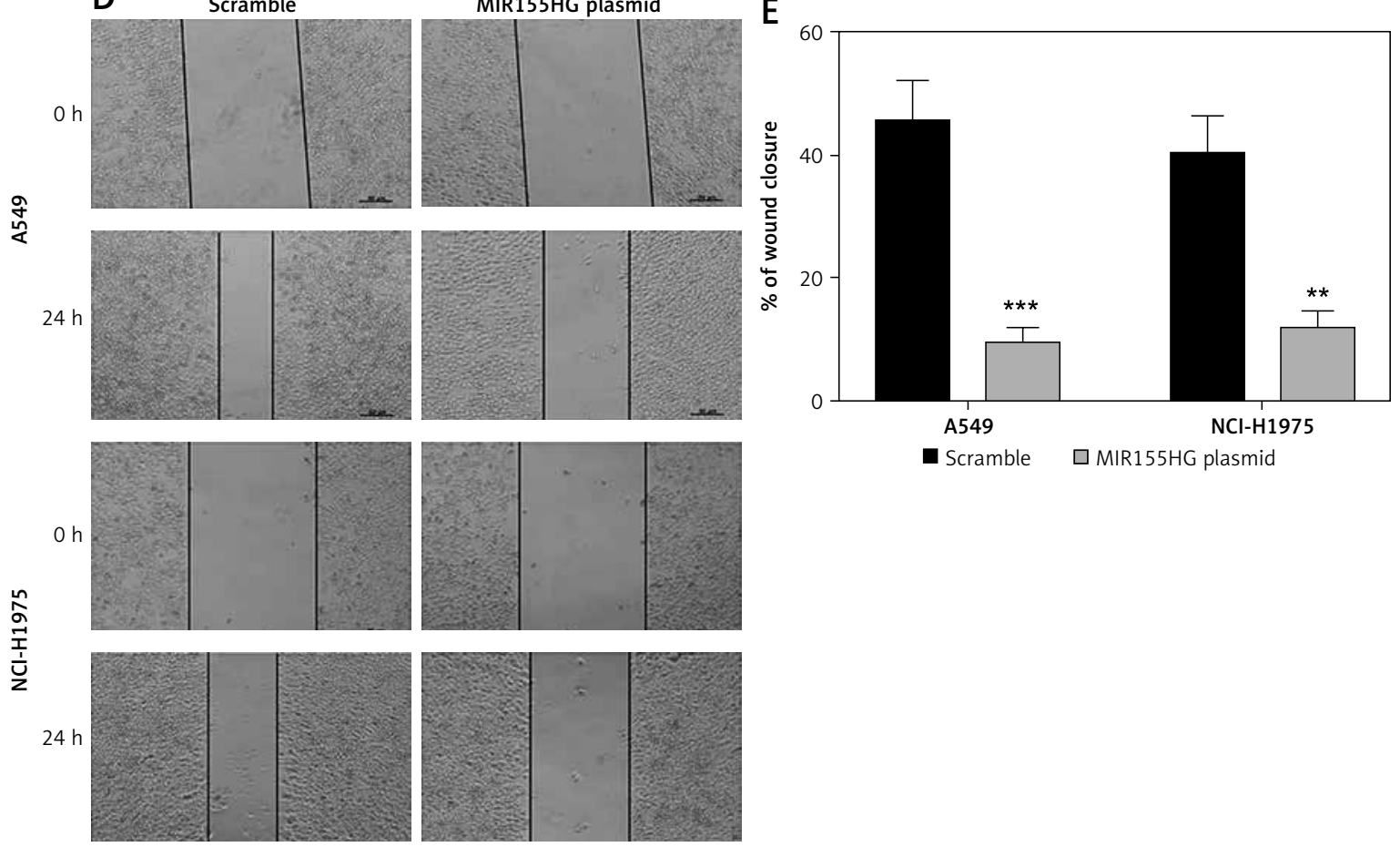

- Scramble $\square$ MIR155HG plasmid

Figure 5. The effect of IncRNA MIR155HG on lung adenocarcinoma cells. A - The expression levels of MIR155HG in LUAD cell lines (NCI-H1299, A549, NCl-H1975) and human normal lung epithelial cells (BEAS-2B) were detected by RT-qPCR. B - The transfection efficiency of MIR155HG plasmid was detected by RT-qPCR. The effects of MIR155HG on the proliferation $(C)$, migration (D, E) 

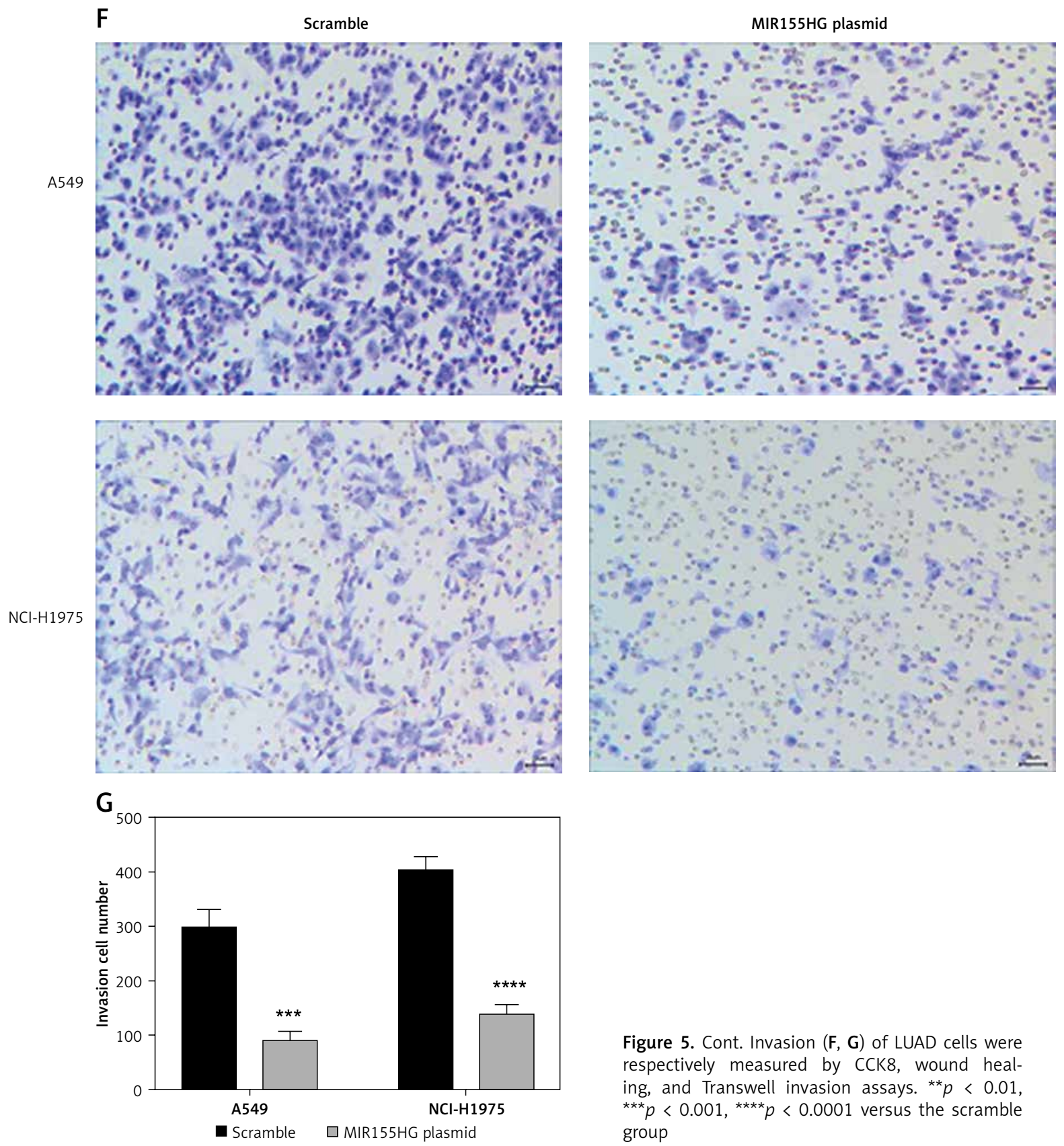

healing assay, and Transwell invasion assay. MIR155HG plasmid obviously enhanced the expression of CD226, but this situation was reversed by the introduction of miR-212-3p mimic (Figures $7 \mathrm{~A}, \mathrm{~B})$. The inhibition of the proliferation of LUAD cells caused by MIR155HG plasmid was changed by the introduction of miR-212-3p mimic (Figure $7 \mathrm{C}$ ). Furthermore, the migration and invasion of the cells were suppressed by MIR155HG plasmid, which was improved in LUAD cells co-transfected with MIR155HG plasmid and miR-212-3p mimic (Figures 7 D-G). Therefore, MIR155HG overexpression inhibited the cellular proliferation, migration, and invasion through sponging miR-21-3p to regulate CD226 expression.

Figure 5. Cont. Invasion (F, G) of LUAD cells were respectively measured by CCK8, wound healing, and Transwell invasion assays. ${ }^{* *} p<0.01$, ${ }^{\star * \star} p<0.001,{ }^{* * * *} p<0.0001$ versus the scramble group

\section{Discussion}

Over half of LUAD cases are diagnosed as advanced metastatic disease with very poor prognosis $[8,10]$. Therefore, the exploration of the pathogenesis mechanism of LUAD is urgent and necessary in order to finding effective diagnoses and treatments. LncRNAs play an important role in many cellular processes such as proliferation, differentiation, and metastasis [15], and the dysregulation of IncRNAs has been closely related to the progression of cancer $[16,17]$. Moreover, IncRNAs can function as ceRNAs to regulate gene expression through sponging miRNAs [14]. Thus, IncRNAs have received more and more atten- 
A MIR155HG: 5'-acacctttgattcaACTGTTA-3'
\[ \||\|\mid\| \]
miR-212-3p: 3'-ccggcacugaccucUGACAAU-5

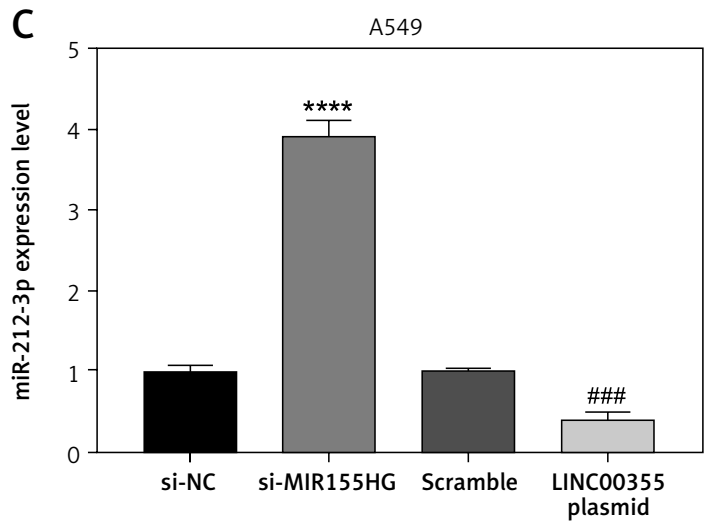

D

miR-212-3p: 3'-ccGGCACUGACCUCUGACAAu-5' CD226: 5'-acCTATGAT T -CTGATTGTTa-3'

miR-212-3p: 3'-ccggcaCUGACC-UCUG-ACAAu-5' CD226: 5'-taataaGATA GGAAGACTTGTTa-3'

miR-212-3p: 3'-ccggcacugaccucUGACAAu-5 CD226: || || || 5'-tcatttttcatagaACTGTTt-3'

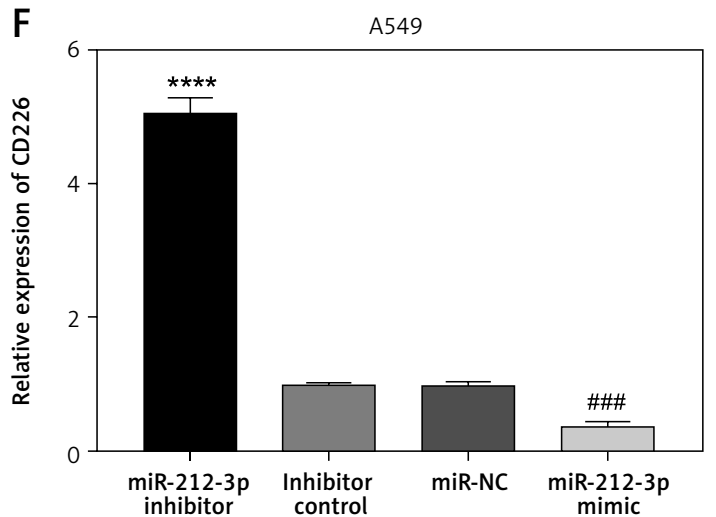

B
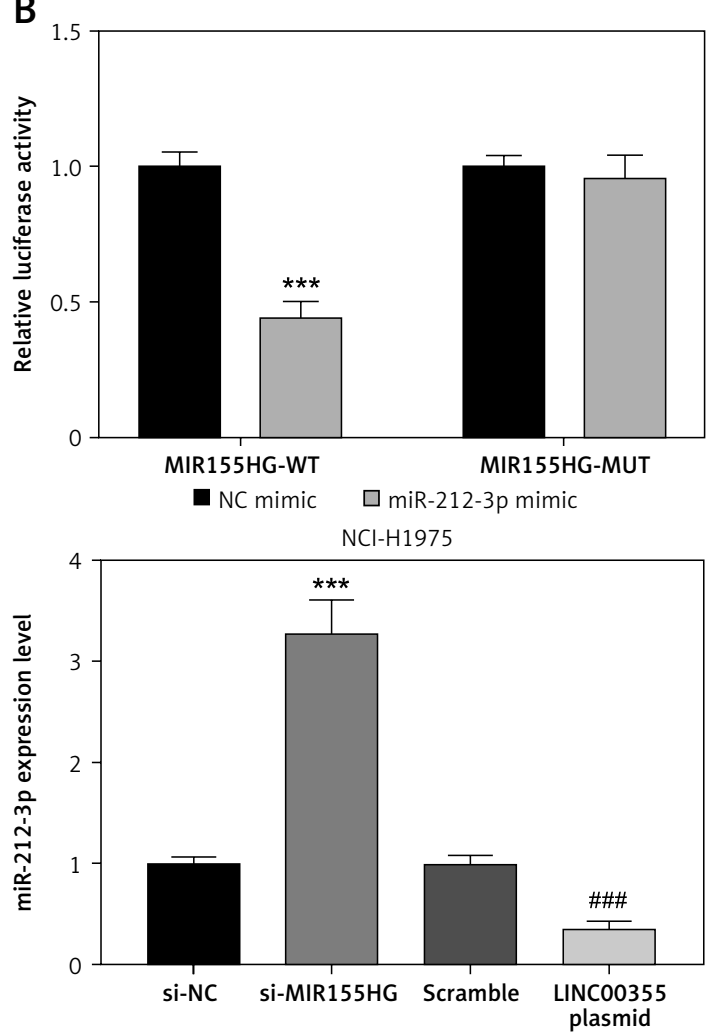

E
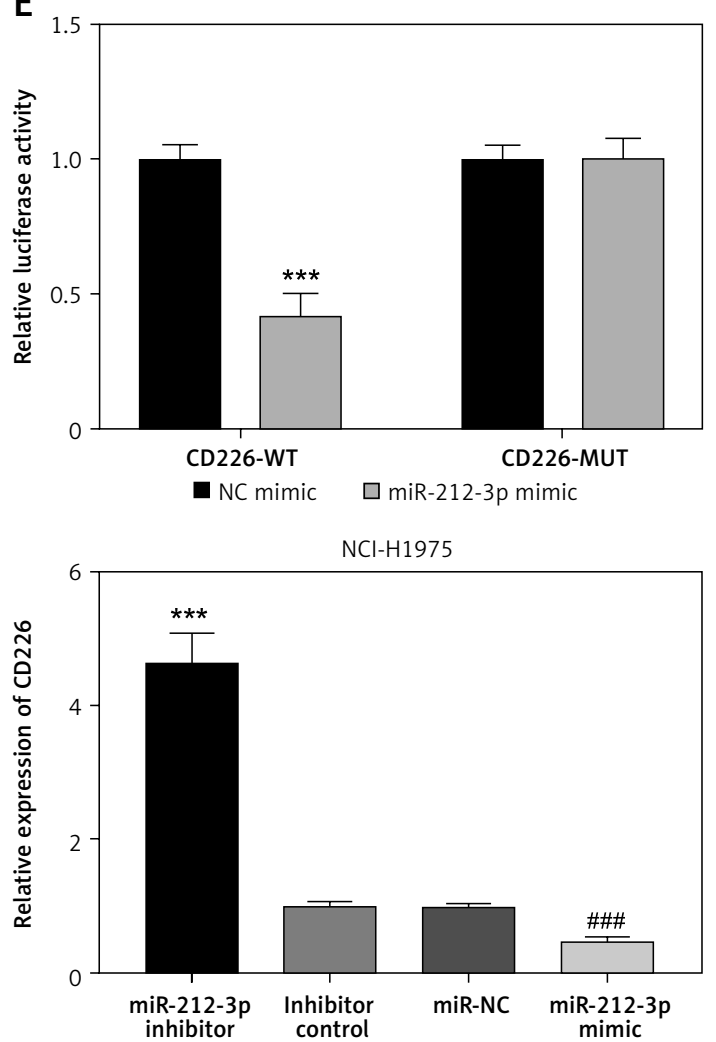

Figure 6. MIR155HG functions as a sponge of miR-212-3p and CD226 is a target of miR-212-3p. A - The binding sites between MIR155HG and miR-212-3p. B - The binding relationship was validated by the dual luciferase reporter gene assay. $\mathbf{C}$ - The expression of miR-212-3p in LUAD cells transfected with MIR155HG plasmid, siRNA MIR155HG (si-MIR155HG), or corresponding controls was detected by RT-qPCR. D - The binding sites between miR-212-3p and CD226. E - The binding relationship was validated by the dual luciferase reporter gene assay. The expression levels of CD226 in LUAD cells transfected with an miR-212-3p mimic, inhibitor, or controls were respectively detected by (F) RT-qPCR 

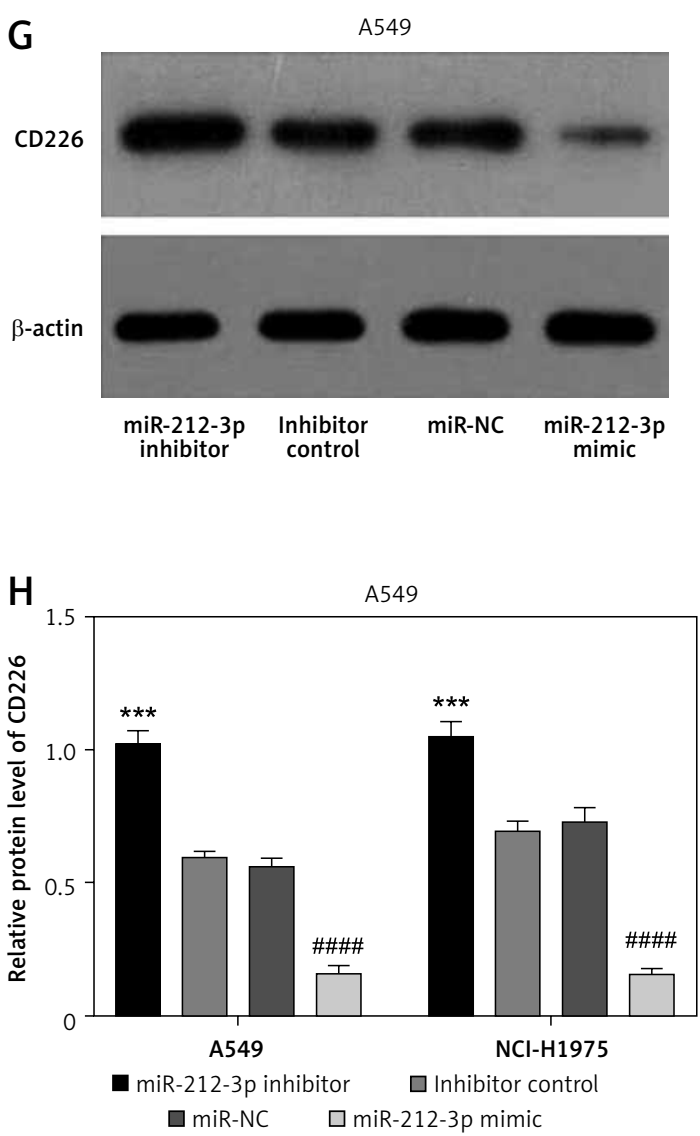

tion. In the present study, we explored IncRNAmiRNA-mRNA ceRNA networks in LUAD using bioinformatics tools and validated the interactions among IncRNA, miRNA, and mRNA by biological experiments.

Based on the TCGA database, we obtained LUAD datasets and divided the patients into two groups (metastasis and non-metastasis groups). Then, we analysed the differential expression of IncRNAs, miRNAs, and mRNAs, and obtained 854 DELs, 150 DEMs, and 2211 DEGs between metastasis and non-metastasis LUAD patients. Combined with the target relationship of DEMs with IncRNAs and mRNAs, we obtained 44 IncRNA-miRNA pairs and 132 miRNA-mRNA pairs, including 15 IncRNAs, 7 miRNAs, and 91 mRNAs, and constructed the IncRNA-miRNA-mRNA ceRNA network. mRNAs in the ceRNA network were enriched through GO and KEGG pathway analysis. For GO terms, the mRNAs were mostly associated with small GTPase-mediated signal transduction, chromosomal region, and chromatin binding. For KEGG pathway analysis, the pathways significantly correlated to LUAD metastasis including p53 signalling pathway and transcriptional misregulation in cancer.

To further screen the ceRNA network, we downloaded the prognosis-related clinical information of the ceRNA network and analysed the OS of each molecule in the ceRNA networks. Combined

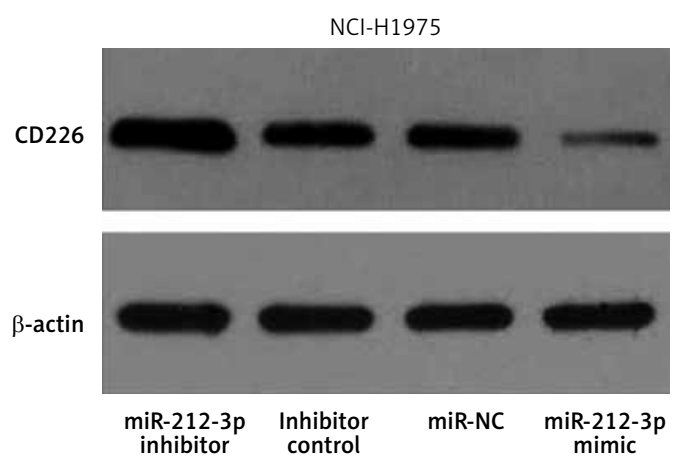

Figure 6. Cont. The expression levels of CD226 in LUAD cells transfected with an miR-212-3p mimic, inhibitor, or controls were respectively detected by (F) RT-qPCR and (G, H) western blot. ${ }^{* * *} p<0.001$, ${ }_{* * *}^{*} p<0.0001$ versus NC mimic, si-NC or inhibitor control; ${ }^{\# \#} p<0.001,{ }^{\# \# \# p} p<0.0001$ versus scramble or miR-NC

with the interaction among IncRNA, miRNA, and mRNA, we obtained 2 IncRNA-miRNA interaction pairs and 4 miRNA-mRNA interaction pairs, including 1 miRNA, 2 IncRNAs, and 4 mRNAs, to construct the prognosis-related ceRNA network. In this network, MIR155HG and ADAMTS9-AS2 can function as ceRNAs of miR-212 to regulate EPM2AIP1, LAX1, PRICKLE2, and CD226. These molecules were associated with OS and could be valuable predictive factors for the prognosis of LUAD patients. For instance, ADAMTS9-AS2 acts as a tumour suppressor or a carcinogenic factor in different cancers [22-24], and ADAMTS9-AS2 has been proven to be a potential predictive biomarker in LUAD and breast cancer $[25,26]$. In addition, the aberrant expression of miR-212-3p has been linked to cell proliferation, adhesion, differentiation, and migration [27].

To validate the roles of the molecules in the prognosis-related ceRNA network in LUAD, we randomly selected MIR155HG, miR-212-3p, and CD226 for in vitro experiments. Firstly, MIR155HG was found to be downregulated in LUAD cells, which was consistent with the bioinformatics results. MIR155HG overexpression inhibited the proliferation, migration, and invasion of LUAD cells. Furthermore, our prognosis analysis indicated that high expression of MIR155HG had a relatively good prognosis compared with its low expression. Thus, MIR155HG 
A

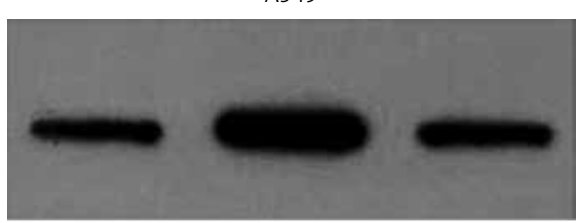

$\beta$-actin

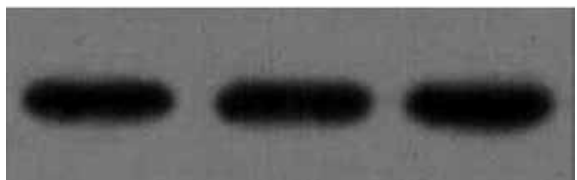

$\operatorname{miR-NC}+$

scramble

miR-NC + miR-212-3p mimic +

miR155HG

B

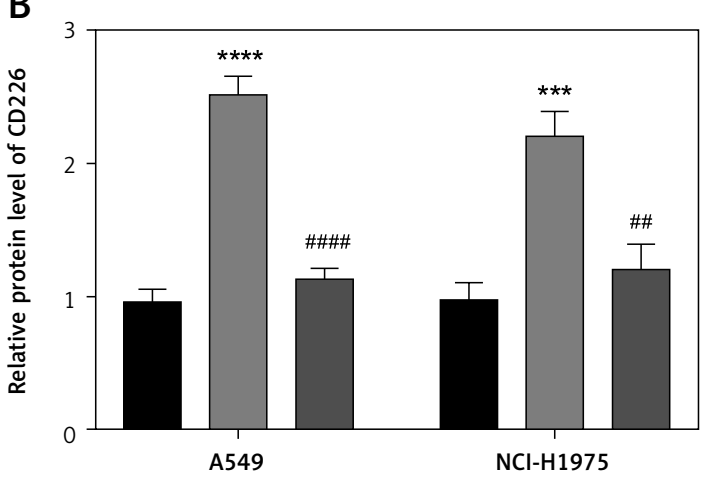

- miR-NC + scramble

$\square$ miR-NC + MIR155HG

$\square$ miR-212-3p mimic + MIR155HG

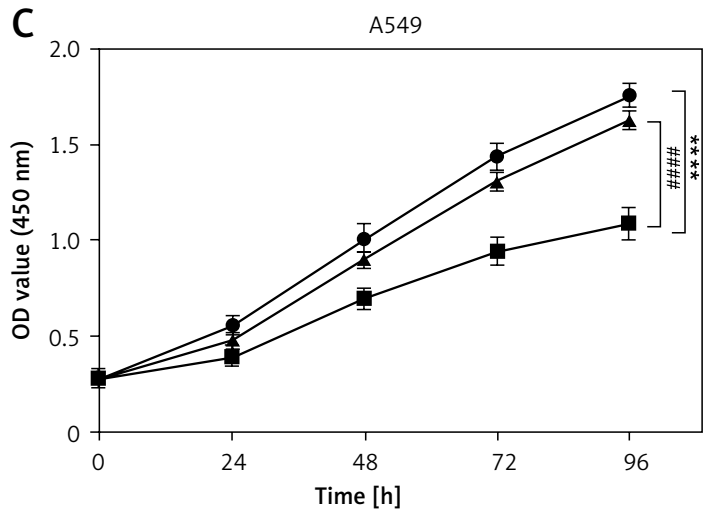

- miR-NC + scramble

- miR-NC + MIR155HG plasmid

$\rightarrow$ miR-212-3p mimic + MIR155HG plasmid

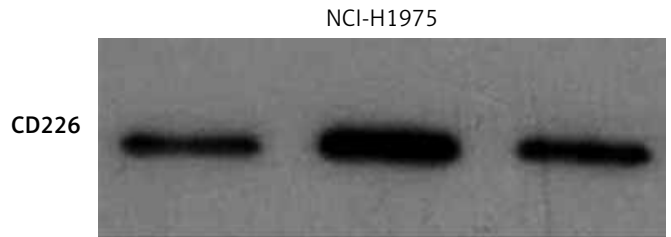

$\beta$-actin

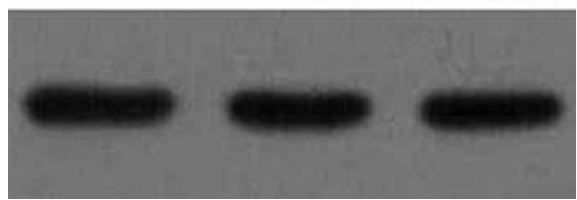

miR-NC +

miR-NC + miR-212-3p mimic + scramble

miR155HG

miR155HG

plasmid

Figure 7. MIR155HG inhibits the growth of lung adenocarcinoma cells through regulation of the miR-212-3p/ CD226 axis. After LUAD cells were transfected with miR-NC+ scramble-miR-NC + MIR155HG plasmid, and miR-212$3 p$ mimic + MIR155HG plasmid, (A, B) the protein expression of CD226 was determined by western blot; (C) the cell proliferation was measured by CCK-8

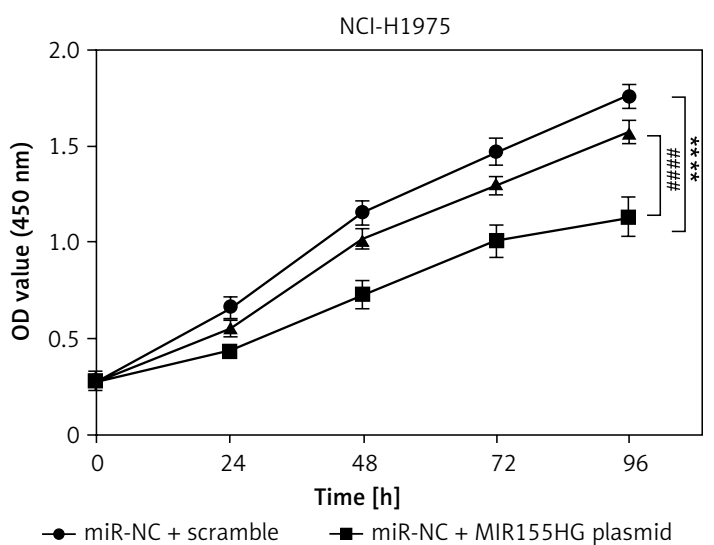

- miR-212-3p mimic + MIR155HG plasmid 
D

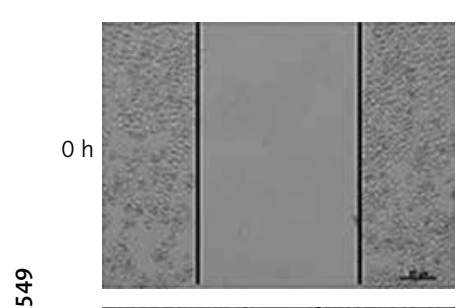

高

$24 \mathrm{~h}$
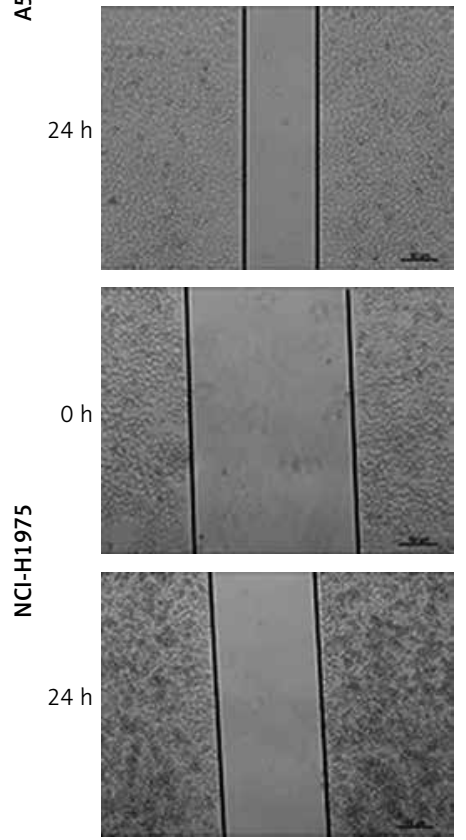

miR-NC + scramble
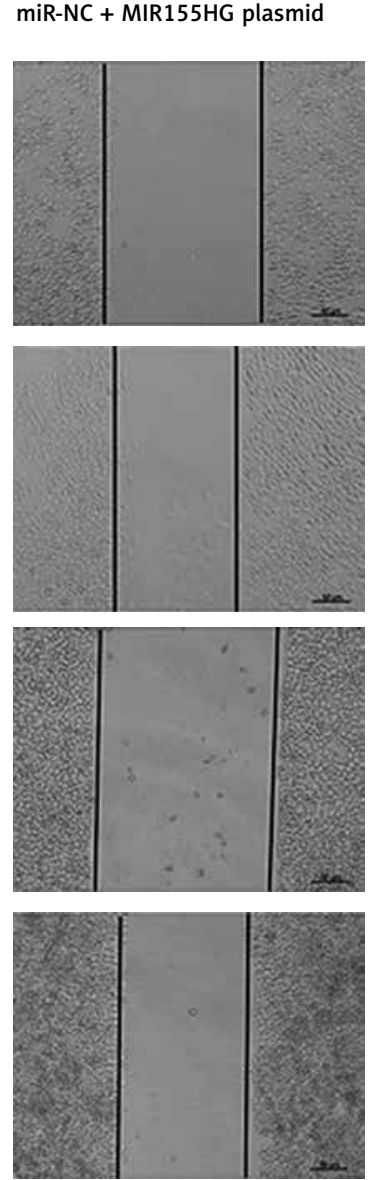

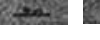

miR-212-3p mimic + MIR155HG plasmid
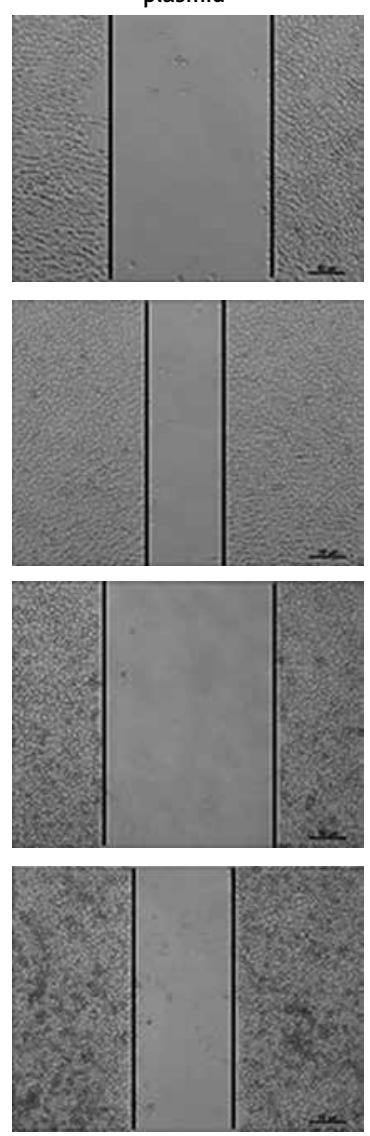

miR-NC + MIR155HG plasmid
E

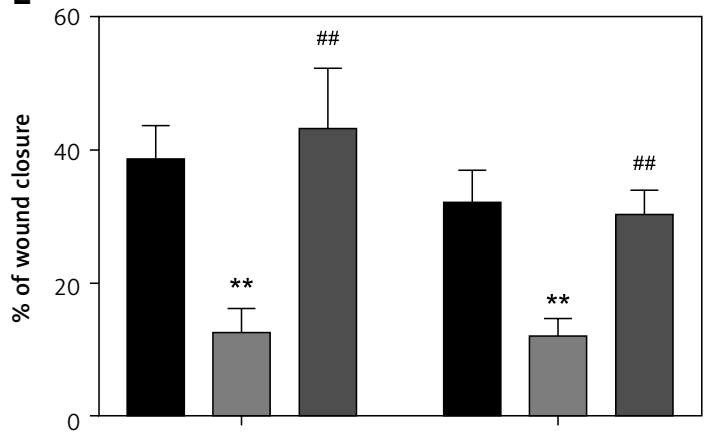

$\mathrm{NCl}-\mathrm{H} 1975$

- miR-NC + scramble $\square$ miR-NC + MIR155HG plasmid

$\square$ miR-212-3p mimic + MIR155HG plasmid

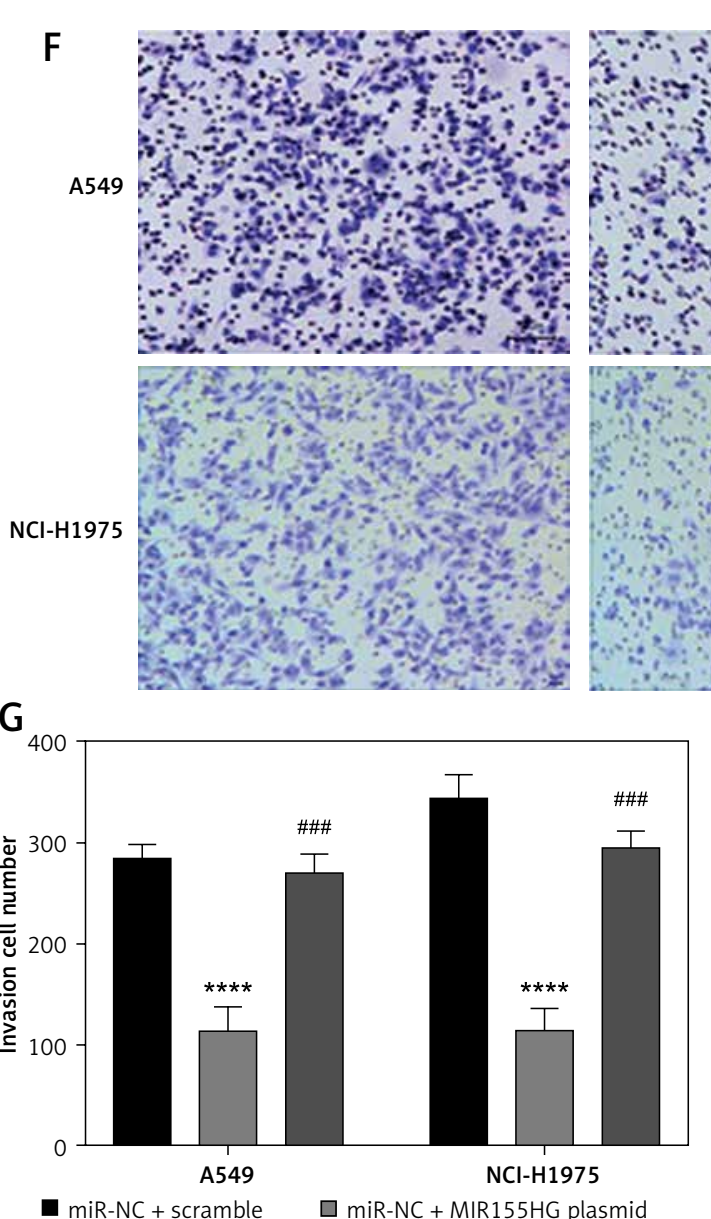

miR-NC + scramble $\square$ miR-NC + MIR155HG plasmid

$\square$ miR-212-3p mimic + MIR155HG plasmid
Figure 7. Cont. The cell (D, E) migration and $(\mathrm{F}, \mathrm{G})$ invasion were respectively detected by wound healing assay and Transwell invasion assay. ${ }^{* * *} p<0.001,{ }^{* * * *} p<0.0001$ versus miR-NC + scramble; ${ }^{\#} p<0.01, \# \#$, $p<0.0001$ versus miR-NC + MIR155HG plasmid
miR-212-3p mimic + MIR155HG plasmid

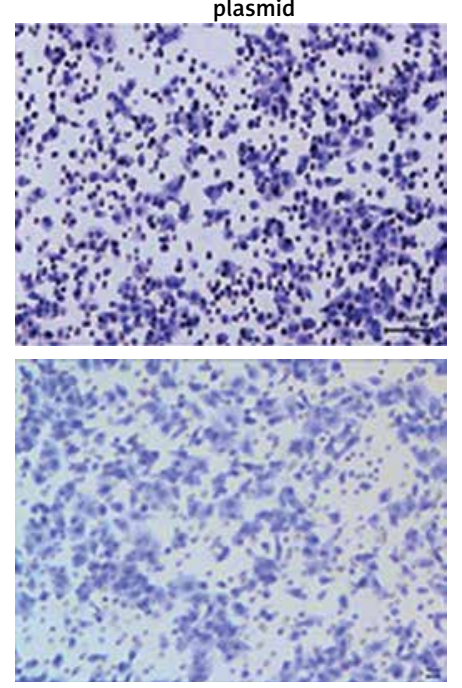


can be considered as a tumour suppressor in LUAD and a potential prognosis biomarker. These findings are in agreement with previous reports. Peng et al. found that a high level of MIR155HG, as a potential prognostic biomarker, was closely correlated with better OS in LUAD, cholangiocarcinoma, and skin cutaneous melanoma [20]. Secondly, our study confirmed that MIR155HG could function as a ceRNA of miR-212-3p, and CD226 was a direct target gene of miR-212-3p, which was consistent with the result of the prognosis-related ceRNA network. Thirdly, the high expression of CD226 induced by MIR155HG overexpression was changed by the introduction of miR-212-3p mimic. In addition, the inhibition of the proliferation, migration, and invasion of LUAD cells caused by MIR155HG overexpression was reversed in LUAD cells co-transfected with MIR155HG plasmid and miR-212-3p mimic. Previous reports suggested that CD226 was involved in tumour immune responses and regulation of the natural killer cell anti-tumour response [28-30]. Moreover, it has been reported that CD226 is critical for the metastasis of lung cancer cells [21]. Collectively, MIR155HG inhibited the proliferation, migration, and invasion of LUAD cells through sponging miR-212-3p to regulate CD226 expression.

In conclusion, our study constructed a prognosis-related ceRNA network to understand the pathogenesis of LUAD. The results indicated that MIR155HG and ADAMTS9-AS2 can function as ceRNAs to sponge miR-212-3p to regulate EPM2AIP1, LAX1, PRICKLE2, and CD226. Furthermore, the interaction among MIR155HG, miR-212-3p, and CD226 was validated by biological experiments, indicating that MIR155HG inhibited the growth of LUAD through the miR-212-3p/CD226 axis. These initial findings urge further experiments to validate the remaining IncRNA-miRNA-mRNA interactions in the ceRNA network.

\section{Conflict of interest}

The authors declare no conflict of interest.

\section{References}

1. Hayasaka K, Shiono S, Matsumura Y, et al. Epidermal growth factor receptor mutation as a risk factor for recurrence in lung adenocarcinoma. Ann Thorac Surg 2018; 105: 1648-54.

2. Avancini A, Sartori G, Gkountakos A, et al. Physical activity and exercise in lung cancer care: will promises be fulfilled? Oncologist 2020; 25: e555-69.

3. Huang HT, Xu YM, Ding SG, et al. The novel IncRNA PTTG3P is downregulated and predicts poor prognosis in non-small cell lung cancer. Arch Med Sci 2020; 16: 931-40.

4. Denisenko TV, Budkevich IN, Zhivotovsky B. Cell deathbased treatment of lung adenocarcinoma. Cell Death Dis 2018; 9: 117.
5. de Groot PM, Wu CC, Carter BW, Munden RF. The epidemiology of lung cancer. Transl Lung Cancer Resh 2018; 7: 220-33.

6. Arnold BN, Thomas DC, Rosen JE, et al. Lung cancer in the very young: treatment and survival in the National Cancer Data Base. J Thorac Oncol 2016; 11: 1121-31.

7. Carnio S, Di Stefano RF, Novello S. Fatigue in lung cancer patients: symptom burden and management of challenges. Lung Cancer 2016; 7: 73-82.

8. Yousem SA. Role of molecular studies in the diagnosis of lung adenocarcinoma. Modern Pathol 2012; 25 Suppl 1: S11-17.

9. Cooke M, Baker MJ, Kazanietz MG. Rac-GEF/Rac signaling and metastatic dissemination in lung cancer. Front Cell Develop Biol 2020; 8: 118.

10. Richardson AM, Havel LS, Koyen AE, et al. Vimentin is required for lung adenocarcinoma metastasis via heterotypic tumor cell-cancer-associated fibroblast interactions during collective invasion. Clin Cancer Res 2018; 24: 420-32.

11. Karreth FA, Pandolfi PP. ceRNA cross-talk in cancer: when ce-bling rivalries go awry. Cancer Discov 2013; 3: 1113-21.

12. Li L, Wei J, Hei J, Ren Y, Li H. Long non-coding RNA H19 regulates proliferation of ovarian granulosa cells via STAT3 in polycystic ovarian syndrome. Arch Med Sci DOI: https://doi.org/10.5114/aoms.2019.89254.

13. Salmena L, Poliseno L, Tay Y, Kats L, Pandolfi PP. A ceRNA hypothesis: the Rosetta Stone of a hidden RNA language? Cell 2011; 146: 353-8.

14. Liang W, Sun F. Competing endogenous RNA Network Analysis Reveals Pivotal ceRNAs in adrenocortical carcinoma. Front Endocrinol 2019; 10: 301.

15. Chan JJ, Tay Y. Noncoding RNA:RNA regulatory networks in cancer. Int J Mol Sci 2018; 19: 1310.

16. Wang J, Ding W, Xu Y, et al. Long non-coding RNA RHPN1-AS1 promotes tumorigenesis and metastasis of ovarian cancer by acting as a ceRNA against miR-596 and upregulating LETM1. Aging 2020; 12: 4558-72.

17. Cai Y, Sheng Z, Chen Y, Wang J. LncRNA HMMR-AS1 promotes proliferation and metastasis of lung adenocarcinoma by regulating MiR-138/sirt6 axis. Aging 2019; 11: 3041-54.

18. Ogunwobi OO, Kumar A. Chemoresistance mediated by ceRNA networks associated with the PVT1 locus. Front Oncol 2019; 9: 834.

19. Li X, Li B, Ran P, Wang L. Identification of ceRNA network based on a RNA-seq shows prognostic IncRNA biomarkers in human lung adenocarcinoma. Oncol Letters 2018; 16: $5697-708$

20. Peng L, Chen Z, Chen Y, Wang X, Tang N. MIR155HG is a prognostic biomarker and associated with immune infiltration and immune checkpoint molecules expression in multiple cancers. Cancer Med 2019; 8: 7161-73.

21. Morimoto K, Satoh-Yamaguchi K, Hamaguchi A, et al. Interaction of cancer cells with platelets mediated by Necl-5/poliovirus receptor enhances cancer cell metastasis to the lungs. Oncogene 2008; 27: 264-73.

22. Wang F, Tang C, Xu D, et al. LncRNA ADAMTS9-AS2 suppresses the proliferation of gastric cancer cells and the tumorigenicity of cancer stem cells through regulating SPOP. J Cell Mol Med 2020; 24: 4830-8.

23. Liu D, Wu K, Yang Y, Zhu D, Zhang C, Zhao S. Long noncoding RNA ADAMTS9-AS2 suppresses the progression of esophageal cancer by mediating $\mathrm{CDH} 3$ promoter methylation. Mol Carcinogen 2020; 59: 32-44.

24. Li Y, Wan Q, Wang W, et al. LncRNA ADAMTS9-AS2 promotes tongue squamous cell carcinoma proliferation, 
migration and EMT via the miR-600/EZH2 axis. Biomed Pharmacother 2019; 112: 108719.

25. Jin D, Song Y, Chen Y, Zhang P. Identification of three IncRNAs as potential predictive biomarkers of lung adenocarcinoma. BioMed Res Int 2020; 2020: 7573689.

26. Deva Magendhra Rao AK, Patel K, Korivi Jyothiraj S, et al. Identification of IncRNAs associated with early-stage breast cancer and their prognostic implications. Mol Oncol 2019; 13: 1342-55.

27. Rong HT, Liu DW. Identification of differentially expressed miRNAs associated with thermal injury in epidermal stem cells based on RNA-sequencing. Exp Ther Med 2020; 19: 2218-28.

28. Fourcade J, Sun Z, Chauvin JM, et al. CD226 opposes TIGIT to disrupt Tregs in melanoma. JCI insight 2018; 3: e121157.

29. Peng YP, Xi CH, Zhu Y, et al. Altered expression of CD226 and CD96 on natural killer cells in patients with pancreatic cancer. Oncotarget 2016; 7: 66586-94.

30. Du X, de Almeida P, Manieri N, et al. CD226 regulates natural killer cell antitumor responses via phosphorylation-mediated inactivation of transcription factor FOXO1. Proc Natl Acad Sci USA 2018; 115: e11731-40. 